\title{
Selection into Identification in Fixed Effects Models, with Application to Head Start
}

\author{
Douglas L. Miller* \\ Cornell University \\ and NBER
}

\author{
Na'ama Shenhav ${ }^{\dagger}$ \\ Dartmouth College \\ and NBER
}

\author{
Michel Grosz $\ddagger$ \\ Federal Trade Commission
}

October $12,2021^{\S}$

\begin{abstract}
Many papers use fixed effects (FE) to identify causal impacts of an intervention. When treatment status only varies within some FE groups (e.g., families, for family fixed effects), FE can induce non-random selection of groups into the identifying sample, which we term selection into identification (SI). This paper empirically documents SI in the context of several family fixed effects (FFE) applications with a binary treatment. We show that the characteristics of the FFE identifying sample are different than the overall sample (and the policy-relevant population), including having larger families. The main implication of this is that when treatment effects are heterogeneous, the $\mathrm{FE}$ estimate may not be representative of the average treatment effect (ATE). We show that a reweighting-on-observables FE estimator can help recover the ATE for policy-relevant populations, and recommend its use either as a primary estimator or as a diagnostic tool to assess the importance of SI. We apply these insights to re-examine the longterm effects of Head Start in the PSID and the CNLSY using FFE. When we reweight the FFE estimates, we find that Head Start leads to a 2.1 percentage point (p.p.) increase (s.e. $=5.9$ p.p.) in the likelihood of attending some college for white Head Start participants in the PSID. This participants' ATE is $83 \%$ smaller than the traditional FFE estimate (12 p.p). We also find that the CNLSY Head Start participants' ATE is smaller than the FE estimates. This raises new concerns with the external validity of $\mathrm{FE}$ estimates.
\end{abstract}

\footnotetext{
${ }^{*}$ Doug Miller, Policy Analysis and Management, Cornell University, Email: dlm336@cornell.edu

${ }^{\dagger}$ Na'ama Shenhav, Department of Economics, Dartmouth College, E-mail: naama.shenhav@dartmouth.edu

${ }^{\ddagger}$ Michel Grosz, Bureau of Economics, Federal Trade Commission, E-mail: mgrosz@ftc.gov

${ }^{\S}$ We would like to thank Colin Cameron, Liz Cascio, Janet Currie, Hilary Hoynes, Pat Kline, Erzo F.P. Luttmer, Jordan Matsudaira, Zhuan Pei, Maya Rossin-Slater, Doug Staiger, Dmitry Taubinsky, Chris Walters, and participants at the AEA Meetings, Cornell, Dartmouth, CSWEP CEMENT Workshop, Hebrew University, Mcgill University, NBER Labor/Children's Summer Institute, Northwestern, SEA Meetings, SOLE, Syracuse/Cornell Summer Workshop in Education and Social Policy, UC Merced, and the War on Poverty Conference at the University of Michigan. We are grateful to Alex Magnuson, Wenran Li, Wenrui Huang, Jack Mueller, and Mary Yilma for excellent research assistance. The views expressed in this article are not necessarily those of the Federal Trade Commission nor any of its commissioners.
} 


\section{Introduction}

Fixed Effects (FE) are frequently used to obtain identification of the causal impact of an attribute, intervention, or policy. These models have been used to identify the impacts of academic peers (school-grade FE; Hoxby, 2000; Carrell and Hoekstra, 2010); criminal peers (facility-offense FE; Bayer, Hjalmarsson and Pozen, 2009); the local health care environment (individual FE; Finkelstein, Gentzkow and Williams, 2016); participation in means-tested programs (family FE; Currie and Thomas, 1995; Garces, Thomas and Currie, 2002; Deming, 2009; Rossin-Slater, 2013); neighborhood quality (family FE; Chetty and Hendren, 2018a); and minimum wage laws (county-pair-year FE; Dube, Lester and Reich, 2010), to give a few examples. Many of the estimates in these studies are targeting the average treatment effect for a policy-relevant population (e.g., participants). However, in contrast with other common estimators, there is not yet a comprehensive framework for considering the external validity of $\mathrm{FE}$ estimates.

In this paper, we document that FE can induce a special type of systematic selection in estimation, which we term "selection into identification" (SI). SI occurs because FE estimates are only identified from FE groups (e.g. families, for family FE) that have variation in treatment ("switchers"), which may exclude some groups. ${ }^{1}$ This is a distinct problem from whether within-group comparisons are internally valid, which has been the typical subject of debate for FE estimators (e.g., Bound and Solon, 1999), and which is not the focus of this paper.

While researchers may be aware of this issue in an abstract sense, our review of the applied literature indicates that it is rarely discussed and almost never empirically examined. This may be because researchers assume that SI is a minor concern, or because there is not a user-friendly tool to address it. We find across multiple previous applications of FE that switchers are typically (i) a small subset of the sample and (ii) systematically different than the overall population. This highlights a new and important concern about the external validity of FE estimates. ${ }^{2}$ We also show that reweighting-on-observables methods can be used to help "undo" SI to recover the average treatment effect (ATE) for policy-relevant populations. We apply these insights to re-examine the external validity of prior family FE (FFE) estimates of the long-run impact of Head Start, a federally-funded pre-school program.

We begin by illustrating SI in a well-known application of FE: an FFE model where the treatment of interest is an indicator for whether an individual attended Head Start (see, e.g., Garces, Thomas and Currie, 2002, henceforth GTC; Deming, 2009). We use the Panel Study of Income Dynamics (PSID), as in GTC. ${ }^{3}$ First, we show that FFE uses substantially fewer identifying groups

\footnotetext{
${ }^{1}$ In the presence of control variables that vary within a group, then there may be variation among non-switchers "net of controls." We focus primarily on cases where this phenomenon is small in magnitude, but include a discussion of this issue in Section 5.

${ }^{2} \mathrm{SI}$ is distinct from issues related to conditional variance weighting in Gibbons, Suarez and Urbancic (2018), which focuses on cases where all groups are switchers.

${ }^{3}$ Similar FFE models have been used to evaluate many other treatments; for public housing, see Andersson et al. (2016); for WIC, see Chorniy, Currie and Sonchak (2018); Currie and Rajani (2015); for health, see Almond, Chay
} 
relative to an estimation model without FE. Among the 5,355 children that have siblings in our sample, only 1,098 children reside in switcher households. Second, we find that the loss of sample variation is systematically related to observables. In particular, families that have a very low likelihood of attending Head Start or a very high likelihood of attending Head Start are both unlikely to be switchers. Additionally, families with more children are significantly more likely to be switchers. As a result, switchers are not representative of the overall sample or Head Start participant families along many dimensions. Third, SI varies across subgroups: the FFE identifying sample misses $93 \%$ of the sibling sample for white children, but only $62 \%$ of the sample for black children.

The main implication of SI is that under heterogeneous treatment effects, the FE estimate is no longer representative of the ATE for the sample. We show that the bias from SI is not addressed by "undoing" conditional variance weighting among switchers (Gibbons, Suarez and Urbancic, 2018), and that in our applications the bias from SI is larger than that from conditional variance weighting. ${ }^{4}$ A second result is that part of the difference between the OLS estimate and FE estimate reflects the disparity in the identifying sample (i.e., the causal treatment effect for non-switchers), and should not be interpreted as solely reflecting OLS bias. ${ }^{5}$

To help recover the ATE for policy-relevant populations ("targets"), we develop a method for reweighting FE estimates that builds on extrapolation techniques from the experimental and IV literatures. ${ }^{6}$ In particular, we show that the ATE for a given target can be computed by weighting group-level FE estimates by the ratio of two propensity scores: (i) the propensity to be in the target (e.g., a program participant) and (ii) the propensity to be in the switcher population. This reweighting is valid under two key assumptions: (1) that these propensity scores can be estimated using observable covariates; and (2) that treatment effects are uncorrelated with being a switcher conditional on covariates. We discuss testable implications of these assumptions and show empirical support for them in our applications. These assumptions provide a weaker alternative to the typical homogeneous treatment effects assumption required for the FE estimate to deliver the ATE. However, in some cases a researcher might prefer the less-externally-valid FE estimate rather than rely on these assumptions. ${ }^{7}$

We demonstrate the performance of our reweighting using Monte Carlo simulations. We find that reweighting reduces or eliminates bias relative to $\mathrm{FE}$ in the presence of covariate-based treat-

and Lee (2005); Figlio et al. (2014); Abrevaya (2006); Black, Devereux and Salvanes (2007); Xie, Chou and Liu (2016), among others. We summarize the prevalence of this design in Section 2.

${ }^{4}$ This is consistent with Gibbons, Suarez and Urbancic (2018), whose findings suggest that the bias from conditional variance weighting is less than $5 \%$ for $75 \%$ of estimates.

${ }^{5}$ Although intuitive, estimating similar OLS coefficients across the overall sample and the switcher sample does not guarantee that the FE estimates are generalizable. This is because OLS bias can vary across covariate values that predict switching. In our application, for example, OLS bias varies with family size (see section 3.3), such that the family-size gradient in treatment effects is much stronger for FE estimates than OLS estimates. As a result, a simple comparison of the OLS coefficients across the OLS and switcher samples will understate the impact of having more large families in the switcher sample.

${ }^{6}$ See Angrist and Fernandez-Val (2013) and Aronow and Carnegie (2013) for extrapolation from IV, and Stuart et al. (2011) and Andrews and Oster (2019) for extrapolation from experiments.

${ }^{7}$ In these cases, researchers can note that FE estimates are specific to the population of switchers, and characterize that population. 
ment heterogeneity. In Section 5, we discuss several extensions of our basic setup, such as how the inclusion of covariates that vary within a group can create additional "residual switchers," and show how reweighting can be applied to a non-linear model.

Based on these findings we propose new standards for practice when presenting results using FE research designs: (i) clearly show the sample size when limited to switcher families and quantify the contribution of "residual switchers"; (ii) show the balance of covariates across switcher and non-switcher families (e.g. Table 2); (iii) reweight FFE estimates for a representative population (e.g. Table 6). Reweighted estimates can be presented either as an additional diagnostic tool or as a primary measure of treatment effects. We are not the first to use the more rigorous reporting standards in (i) and (ii), but in our survey of the FFE literature the vast majority do not discuss either of these issues. Out of 35 papers with binary treatments, only one-third reported the sample sizes we recommend in (i), and just one paper included (ii). ${ }^{8}$

In Section 6, we apply these insights to quantify the importance of selection into identification for FFE estimates of the long-run impact of Head Start. Head Start has a budget of $\$ 8.6$ billion dollars and annually enrolls roughly $60 \%$ of the number of 3 and 4 year old children in poverty, which makes it a quantitatively important intervention for this population (Carneiro and Ginja, 2014). ${ }^{9}$ FFE have been used to identify the long term impacts of Head Start in many of the foundational studies of this program (Currie and Thomas, 1995; Deming, 2009, GTC), which find positive impacts on economic and non-cognitive outcomes of participants measured in adulthood.

First, using data from the PSID and the Children of the National Longitudinal Study of Youth (CNLSY) (as in GTC and Deming, 2009), ${ }^{10}$ we newly document that Head Start has greater returns in larger families across multiple human capital measures. This might result from the fact that parental time investment in children's human capital is spread more thinly in larger families, which in turn could lead to greater returns to alternative investments, such as Head Start. ${ }^{11}$

Second, we show that the FFE estimate of the impact of Head Start is typically too large relative to the reweighted ATE for participants, in part due to over-representation of larger families. Using the PSID, we obtain an FFE estimate that suggests that Head Start leads to a statistically significant 12 p.p. increase in attendance of some college for white individuals. However, when we reweight the FFE estimates to obtain the ATE for white Head Start participants, we find that Head

\footnotetext{
${ }^{8}$ Important exceptions include Finkelstein, Gentzkow and Williams (2016) and Wiswall (2013), who include a substantive discussion of external validity concerns, and Currie and Rossin-Slater (2013), who show the change in summary statistics when moving from the sample of mothers with multiple births to the sample that had at least one hurricane during pregnancy, and are therefore likely to be "switchers." GTC report the number of identifying observations used to identify Head Start for the entire sample (not for subsamples), and Deming (2009) reports the aggregate number of identifying observations used to identify the pre-school, Head Start, and no-formal-care coefficients, but not for each coefficient.

${ }^{9}$ See Gibbs, Ludwig and Miller (2013) for an overview of the Head Start program.

${ }^{10}$ Although we rely on the PSID as in GTC, we use a sample of siblings three times as large as in GTC due to the additional data that has been collected since the publication of that paper and somewhat different inclusion criteria.

${ }^{11}$ In Section 6, we show that this heterogeneity by family size is not explained by other covariates or by larger families having longer sibling cohort spans. Instead it appears that there is something important about family size per se.
} 
Start leads to a 2.1 percentage point (p.p.) increase in the likelihood of attending some college (s.e. $=5.9$ p.p.). This reweighted ATE for participants is $83 \%$ smaller than the FFE estimate ( $p<0.05$ for the difference.) The participants' ATE is also $93 \%$ smaller than the estimated effects on college-going in GTC; $45 \%$ to $91 \%$ smaller than unadjusted estimates for all participants from other FFE studies (Bauer and Schanzenbach, 2016; Deming, 2009); and 51\% smaller than estimates from the county roll-out of Head Start (Bailey, Sun and Timpe, 2018).

Similarly, in the CNLSY, the FFE estimate suggests that Head Start leads to an 8.5 p.p. increase in high school completion, while the reweighted estimate for Head Start participants is $44 \%$ smaller and not statistically significant ( $\mathrm{p}<0.10$ for the difference.) Reweighting also attenuates the previously-estimated impact of Head Start on idleness and having a learning disability, relative to the FFE estimates. In contrast, reweighting has little to no impact on the estimates for the poor health outcome.

In Section 7, we discuss the broader relevance of SI for panel fixed effects models, particularly for short panels and "lumpy" treatment variables (e.g. binary treatments). In particular, we walk through how the guidelines that we have put forth could apply to three prior FE applications.

This paper makes three main contributions. First, we empirically document across multiple FE settings that many groups are not switchers, and that switchers are observably different than non-switchers. ${ }^{12}$ This stands in contrast to the assumption that all FE groups are switchers, which underlies several econometric techniques (Bates et al., 2014; Loken, Mogstad and Wiswall, 2012; Gibbons, Suarez and Urbancic, 2018). It also implies that the method in Gibbons, Suarez and Urbancic (2018) for recovering the ATE from FE models by "undoing" the conditional variance weighting - which relies on this assumption - is not sufficient to recover the ATE in many contexts. Finally, it suggests that more researchers using FE models should examine SI and discuss this in their interpretation of FE estimates. We show that this is rarely done in current practice in Section 2 , and provide easy rules-of-thumb to guide such investigations.

Second, we develop a general reweighting method to obtain the ATE for policy-relevant populations from FE models. ${ }^{13}$ This provides an alternative to defining the population of interest to be switchers, as discussed in Angrist (1998) (for OLS models) and Imai and Kim (2019) (for FE models). In doing so, we add to a small set of papers that provide methods for researchers to extrapolate FE treatment effects beyond switchers. Existing approaches include bounding methods for nonparametric panel models (Section 4 of Chernozhukov et al., 2013), and using a random coefficients model for treatment effect heterogeneity (Wooldridge, 2005, 2019; Bates et al., 2014). Our literature review suggests that practitioners do not pursue either of these current methods for correction. Relative to these other correction methods, we provide easy-to-implement guidelines

\footnotetext{
${ }^{12}$ This is distinct from recent works on the validity of difference-in-difference and other two-way FE strategies, where the empirical specification ensures that SI is unlikely to be a concern. See, e.g. Goodman-Bacon (2018); Borusyak and Jaravel (2017); Callaway and Sant'Anna (2018); Chaisemartin and D'Haultfoeille (2019).

${ }^{13}$ This is distinct from strategies that use reweighting for internal validity, such as traditional propensity score estimation methods.
} 
for using our reweighting approach that is closely fitted to current empirical practice.

Our reweighting solution is most similar in spirit to Angrist and Fernandez-Val (2013), who reweight IV estimates using discrete covariates. ${ }^{14}$ We tailor this idea to the FE context to develop our reweighting method, and show the set of assumptions under which these weights can recover the ATE from FE estimates. Also different than Angrist and Fernandez-Val (2013), we reweight using propensity scores, which allow for greater flexibility in conditioning variables.

Third, our empirical findings contribute to a growing body of work investigating the long term effects of Head Start using quasi-experimental methods (Ludwig and Miller, 2007; Carneiro and Ginja, 2014; Thompson, 2017; Bauer and Schanzenbach, 2016; Johnson and Jackson, 2017; Bailey, Sun and Timpe, 2018; Pages et al., 2019; Barr and Gibbs, 2018; Leuven and de Haan, 2016, in addition to the FFE papers above). These studies typically present LATE or ITT estimates, and find improvements in childhood health, reductions in adolescent behavioral problems and obesity, and increases in adult educational attainment and earnings. ${ }^{15}$ Relative to most of these studies, we evaluate the effect of Head Start on longer-run outcomes; show that these effects vary significantly by family size; and also adjust estimates using covariate re-weighting to get closer to the ATE for Head Start participants. We show that incorporating this adjustment lowers the estimated long term effect of Head Start relative to FE estimates.

\section{A Survey of FFE Applications}

We begin by performing a survey of FE papers in the literature to gauge the prevalence of these methods, particularly among studies that use binary treatments or have small sample sizes (which may be more susceptible to SI.) Since our application focuses on a FFE model, we focus on applications of this particular method in the literature. This focus will lead us to undercount the prevalence of FE more broadly, but provides an unambiguous example of a short-panel setting which is susceptible to SI concerns. We surveyed publications from January 2000 to May 2017 in 11 leading journals that publish applied microeconomics articles. We include all studies that use family fixed effects as a primary or secondary strategy. ${ }^{16}$

Our literature review yields 55 papers published from 2002 to 2017. We provide descriptive

\footnotetext{
${ }^{14}$ More broadly, we relate to a number of studies that use reweighting to account for the discrepancy between "what you want" and "what you get" from common estimators. These include Lochner and Moretti (2015), who reweight OLS with IV weights for greater comparability; Sloczynski (2018), who reweights OLS to obtain the ATE; and Stuart et al. (2011) and Andrews and Oster (2019) who propose reweighting experiments to account for selection into participation.

${ }^{15}$ One exception to this is Pages et al. (2019), who suggest that the effect of Head Start may be negative for recent cohorts, although the identifying sample is not discussed.

${ }^{16}$ We surveyed: AEJ: Applied Economics, AEJ: Economic Policy, AER, AER P\&P, Journal of Health Economics, Journal of Human Resources, Journal of Labor Economics, Journal of Political Economy, Journal of Public Economics, QJE, Review of Economics and Statistics. To identify these articles, we used the search terms "family," "withinfamily," "sibling," "twin," "mother," "father," "brother," "sister," "fixed effect," "fixed-effect," and "birthweight" using queries on journal websites. We then searched within articles to see whether FFE was used in the analysis. Finally, we added some additional papers to the list that we are aware of and did not satisfy these search terms. The resulting list is fairly comprehensive, but still likely to be a slight undercount of FFE articles in these journals.
} 
statistics of these articles in Table 1, including statistics by journal. Overall, these articles account for between 0.5 and 1 percent of the papers published in our sample of journals in any given year, but this varies from 0 to 9 percent of each journal in a given year. The first panel tabulates the frequency of binary treatments and binary outcomes across the sample of papers, the focus of our methodological insights. Nearly two-thirds (35) of the papers have a binary treatment of interest and 23 have a binary outcome. The second and third panels show the varied topics that appear in the sample, spanning health, public, education, and labor fields.

The final panel of the table summarizes the distribution of sample sizes used with FFE. The samples are frequently not limited to families with variation in the treatment variable; therefore, the sample size in the table is an upper bound on the number of observations used for identification. The median number of sibling observations is 6,315 , or roughly $85 \%$ of the sample in our analysis. We note that there is a high variance in sample size across samples, indicating that there is not a threshold for FFE analyses. The bottom $25 \%$ of papers have fewer than 1,200 observations, while the top $25 \%$ have over 160,000 sibling observations.

Appendix Figure B.1 illustrates the popularity of this estimation strategy over time. It shows a steady stream of FFE papers over the past 15 years; and that these papers have an impact on the literature, with a mean 233 citations per article (Google Scholar citations as of May 2019). Moreover, since the survey was completed, additional FFE studies have been published (see e.g. Chetty and Hendren $(2018 a, b))$. We discuss the prevalence of switcher counts in the next section.

\section{$3 \quad$ Fixed Effects and Selection into Identification}

We use a FE research design in our application to address the concern that Head Start treatment may be correlated with some fixed characteristics of a family that also determine outcomes. For example, the decision to participate in Head Start of siblings is influenced by low parental income (a requirement for eligibility), which may independently influence long-term outcomes. As a result, the cross-sectional estimate of the effect of treatment is likely to be biased.

To formalize our setting, let $D_{i} \in\{0,1\}$ indicate whether an individual $i$ participates in treatment (e.g. Head Start) and $g(i)$ be the relevant group (e.g. family) for $i$ of the set of groups $G$ in the sample, and let potential outcomes in the untreated and treated states be $Y_{i}(0), Y_{i}(1)$, respectively. We observe for each $i$ one outcome, $Y_{i}=Y_{i}\left(D_{i}\right)$, treatment, $D_{i}$, and group membership, $g(i)$. For brevity, we will frequently write this simply as $g$. We refer to groups for whom realized $\operatorname{Var}\left(D_{i} \mid i \in g(i)\right)>0$ as "switchers," and denote switching status with a binary variable $S_{g}=1$, and the set of switchers as $G_{S} \subseteq G$.

We assume that treatment may be correlated with group characteristics, e.g. mean family income, but is randomly assigned within groups:

\section{Assumption 1 (Group ID Conditional Independence):}

$$
Y_{i}(0), Y_{i}(1) \Perp D_{i} \mid g(i)=g
$$


Assumption 1 encompasses the standard FE specification assumpton in linear models. It rules out Roy (1951)-type selection into treatment within groups, in which the probability of receiving treatment is correlated with treatment effects, $Y_{i}(1)-Y_{i}(0) \cdot{ }^{17}$ In the context of Head Start, within-family variation in treatment has been shown to be uncorrelated with most observable characteristics of children (Deming, 2009, GTC, 2002), suggesting the assumption is reasonable.

Under this assumption, estimated treament effects $\hat{\delta}_{g, F E}$ are an unbiased (but very noisy) estimate of group-level treatment effects, $\delta_{g} \equiv \mathbb{E}\left[Y_{i}(1)-Y_{i}(0) \mid g(i)=g\right]$. The FE estimate averages $\hat{\delta}_{g, F E}$ for the $g \in G_{S}$, using weights that are proportional to the within-group variance of $D_{i}$ and the number of observations in $g, n_{g}$ (Angrist, 1998; Angrist and Pischke, 2009, eqn. 3.3.7). The FE estimand is:

$$
\delta_{F E}=E_{g}\left[\delta_{g} \cdot \omega_{g, F E}\right]
$$

where

$$
\omega_{g, F E}=\frac{\operatorname{Var}\left(D_{i} \mid g(i)=g, S_{g}=1\right) \cdot n_{g} \cdot \operatorname{Pr}\left(S_{g}=1 \mid g(i)=g\right)}{\sum_{g}\left[\operatorname{Var}\left(D_{i} \mid g(i)=g, S_{g}=1\right) \cdot n_{g} \cdot \operatorname{Pr}\left(S_{g}=1 \mid g(i)=g\right)\right]}
$$

We examine two methodological issues that arise from the FE research design: (i) reduction in identifying variation moving from $G$ to $G_{S}$; and (ii) a change in the composition of the identifying sample due to the fact that $\operatorname{Pr}\left(S_{g}=1 \mid g(i)=g\right)$ is not likely to be the same for all groups. Issue (i) is well understood in principle, but the degree to which $G_{S}$ is smaller than $G$ is often underappreciated, and implicitly assumed to be negligible in many theoretical results. Issue (ii) is more novel, and should cause researchers to update the interpretation of the population for which these estimates are relevant. In our literature review, both of these issues are not reported in empirical practice: one-third of papers with a binary independent variable reported the number of switchers, and one paper showed summary statistics by switching status. ${ }^{18}$

\subsection{Empirical Relevance}

To illustrate these two methodological issues, we use the data from our empirical application, which is described in detail in Section 6. The sample consists of 2986 white children born in the years 1954-1987. The regression of interest estimates the effect of ever having attended Head Start on a dummy for ever having attended college. The coefficient on Head Start in a cross-section regression is 0.049 (s.e. $=0.044$ ). When FFE are added, the coefficient becomes 0.120 (s.e. = 0.053). This result indicates that the impact of Head Start participation on college attendance is

\footnotetext{
${ }^{17}$ Some recent FE strategies explore relaxation of this assumption. For example, in a two-period person-level FE design, Lemieux (1998) estimates union wage returns to both observed and unobserved skills. This approach is extended (with application to farmer adoption of HYV seeds) in Suri (2011) and Verdier and Castro (2019).

${ }^{18}$ We also note that the goal of the latter comparison was to show that within-family comparisons were more valid than cross-sectional comparisons, not to discuss external validity. Even if we look at the more recent period after 2010 , just 5 out of 20 articles reported the number of switchers, suggesting that researchers still do this rarely.
} 
meaningful in magnitude, and statistically significantly different from zero.

We illustrate the identifying variation for the FFE regression of some college on Head Start attendance in Panel (a) of Figure 1, which shows a scatterplot of the deviation in Head Start attendance for each individual $i$ from the mean attendance in his or her family, $g(i)$, HeadStart $i^{-}$ $\overline{\text { HeadStart }_{g(i)}}$, against the within-family deviation in attainment of some college for the sample, AnyCollege $_{i}-\overline{\text { AnyCollege }_{g(i)}} \cdot{ }^{19}$ Strikingly, the largest mass of observations is at $(0,0)$ : the majority of families have no variation in Head Start participation and no variation in the college attendance of their children. Individuals in families with no variation in Head Start account for $96 \%$ of the sample - removing these leaves us with 213 individuals in switching families.

This reduction in identifying observations could result in a selected sample if switching is correlated with family characteristics. To gain intuition about which variables might determine switching we build a simple model of the Head Start participation decision within families. If the probability of attending Head Start is a constant, $\pi$, and independent across siblings in a family, then the probability of switching, $\operatorname{Pr}\left(S_{g}=1\right)$ is simply a function of $\pi$ and family size, $n_{g}$ :

$$
\operatorname{Pr}\left(S_{g}=1\right)=1-(1-\pi)^{n_{g}}-\pi^{n_{g}}
$$

According to this formula, the probability of switching has an inverse-U-shaped relationship with $\pi$, peaking at $\pi=0.5$. Further, for a given level of $\pi$, the likelihood of being in a switching family is increasing with family size. We illustrate these features in Appendix Figure B.2.

The markers in Figure 2 show the actual probability of attending Head Start and of being in a switching family for each family size by black/white race and by whether the mom has some college or not. As in the stylized model, the likelihood of switching is increasing with family size for each of these subgroups. ${ }^{20}$ This could reflect the fact that over time, across children, parents are more likely to be exposed to the program, or are more likely to experience a change in family income, which alters eligibility for the program.

We also observe that switching increases with $\pi$, following the inverse-U. The probability of Head Start attendance among black families and families with low-educated moms is much higher and closer to 0.5, compared to white families and families with high-educated moms; and the switching probability is correspondingly larger for black and low-educated families. As a result, the sample used for FFE identification is comprised of $7 \%$ of the sibling sample for whites, and $38 \%$ of the sibling sample for blacks. Note that while we are focusing on race and maternal education, this notion can be generalized to any other family characteristic, such as SES, that determine $\pi .^{21}$

\footnotetext{
${ }^{19}$ The size of each symbol is weighted by the number of individuals. A value of 0.5 along the horizontal axis, for example, means that a person went to Head Start in a family where half the children attended Head Start. Values other than 0.5 and -0.5 indicate that the share of children that attended Head Start was different than 0.5; e.g. a value of -0.75 means that a person did not go to Head Start in a family where three quarters of the children did.

${ }^{20}$ Appendix Table B.1 shows that this pattern is driven by a much larger incidence of no Head Start participation among smaller families. For example, $78 \%$ of 2 -child families have no Head Start participants, compared with $48 \%$ of families with 5 or more children.

${ }^{21}$ In Appendix Figure B.3, we create a more detailed version of this figure by graphing the share switching by family
} 
This pattern is not unique to the PSID or to Head Start. Panels (b) and (c) of Figure 2 show this relationship using data from two other FFE papers, Collins and Wanamaker (2014) and Deming (2009). In both papers, the treatment variable of interest is binary; migration to the North and Head Start participation, respectively. In each of these samples, the probability of being a switcher is increasing in family size.

\subsubsection{Selection into Identification Driven by Many Variables}

Since SI is likely to affect the balance of characteristics other than family size, we now examine a large number of observable characteristics of switcher families and non-switcher families. Panel A of Table 2 indicates that in addition to having a larger family size, children in switcher families tend to have parents with significantly less education than children in non-switcher families (column 3). These differences in parental education are significant (at the 10 percent level) even in a regression framework where we control for differences in family size and the other covariates in the table (columns 4 and 5). Family income during preschool of children in switcher families is also significantly lower than non-switcher families overall. ${ }^{22}$ These patterns are consistent with switching increasing with the probability of Head Start participation.

Next, we examine a one-dimensional summary of how much overlap there is in the characteristics of switchers and non-switchers. We do so by comparing propensity-score-type weights across switchers and non-switchers. This is in the spirit of the literature on using propensity score to extrapolate experimental results to broader populations (Stuart et al., 2011). For comparison we use propensity-score-derived weights, $\frac{\operatorname{Pr}\left(S_{g(i)}=1 \mid \mathbf{X}_{\mathbf{i g}}\right)}{\operatorname{Pr}\left(\text { HeadStart }_{i}=1 \mid \mathbf{X}_{\mathbf{i g}}\right)}$, which we discuss in more detail in Section 4. These weights give a measure of how aligned the characteristics (vector $\mathbf{X}_{\mathbf{i g}}$ ) of switchers are with the characteristics of Head Start participants, the population of interest. An average value of 1 implies perfect alignment, while a higher value implies that the characteristics of switchers are over-represented relative to the characteristics of Head Start participants. We estimate the elements of this ratio using a multinomial logit.

Panel B of Table 2 shows that this measure is between 1.9 and 3 for the switchers sample, which is at least 0.6 SD larger than for non-switchers. This indicates that the observables of switchers are not aligned with our population of interest, and that this misalignment is worse for switchers than non-switchers ${ }^{23}$.

size against the predicted probability of attending Head Start. It shows that the level of switching is lower than in the theoretical model, suggesting that decisions to attend Head Start are not truly independent across children. However, the general metaphor holds: switching increases with pr(Head Start) and with family size, even if the magnitude is smaller.

${ }^{22}$ If we limit ourselves to families with Head Start participants, we continue to find many statistically significant differences (see Appendix Table B.2.

${ }^{23}$ For example, Stuart et al. (2011) suggest that a 0.1 to $0.25 \mathrm{SD}$ difference in propensity scores between the experimental and non-experimental population may be too large to rely on extrapolation without further adjustments. 


\subsection{Consequences for Estimation: Effective Number of Identifying Observa- tions}

A convenient way to summarize the amount of variation used in FE is by the number of individuals in switching families. However, since not all switchers provide the same amount of identifying variation, this can be a misleading measure. For example, a 4-sibling family with 1 treated and 3 untreated individuals has an $\omega_{g, F E}$ that is $25 \%$ smaller than the $\omega_{g, F E}$ of a family with 2 treated and 2 untreated $(0.25 \cdot 0.75=0.1875<0.25=0.5 \cdot 0.5)$.

We develop a formula for the "effective number of observations," which captures this idea by (i) quantifying the total amount of identifying variation and (ii) converting this into standardized units (person-equivalents).

$$
N_{e f f}=\frac{\sum_{g \in G_{S}} \operatorname{Var}\left(D_{i} \mid g(i)=g\right) \cdot\left(n_{g}-1\right)}{\operatorname{Var}\left(D_{i, \text { reference }}\right)}
$$

The numerator quantifies the "total amount of variation" identifying $\delta_{F E}$ by summing over the variation contributed by each group. This expression is similar to the weight given to each group in the FE estimate, except family size is adjusted for the fact that group-level fixed effects remove one degree of information from each family, $\left(n_{g}-1\right)$. The denominator provides a translation from "total variation" to "person-equivalents" of variation by normalizing by the variation contributed by an individual observation in a fixed, researcher-determined group, $\operatorname{Var}\left(D_{i, \text { reference }}\right)$.

In our application, we report effective observations using two different reference groups. First, we normalize by the variation across units in a cross section regression after controlling for reasonable g-level covariates, $\operatorname{Var}\left(D_{i, \text { reference }}\right)$. This gives the equivalent number of cross-sectional units that would produce the variation used in the FE regression. Second, we normalize by the within-family variation from units in two-unit groups. The difference between this and the number of individuals in switching families informs us about whether the presence of larger groups contributes additional identifying variation. ${ }^{24}$

\subsection{Consequences for Estimation: OLS weights and FE weights}

Under homogeneous treatment effects $\left(\delta_{g}=\delta\right)$, SI has no effect on expected bias in estimation of Equation 2, and the FE estimate trivially is unbiased for the ATE for the sample and the population. There is only a loss of precision that accompanies the overall reduction in sample size.

The more interesting case is when treatment effects are heterogeneous. In that case, SI will lead the FE estimate to provide a biased estimate of the ATE, even if one corrects for the conditional variance weighting of $\mathrm{FE}$ among switchers. To be concrete, let $Z$ be a discrete covariate that varies at the group level, such as family size, that determines the magnitude of the effect of treatment.

\footnotetext{
${ }^{24} \operatorname{Var}\left(D_{i} \mid g(i)=g\right)$ is calculated using the population formula for variance, $\operatorname{Var}\left(D_{i} \mid g(i)=g\right)=$ $\frac{1}{n_{g}} \sum_{i \in g}\left(D_{i}-\frac{\sum_{i \in g} \mathbf{1}\left(D_{i}=1\right)}{n_{g}}\right)^{2}$, rather than the sample formula (which would divide by $\left.n_{g}-1\right)$.
} 
We allow for a different treatment effect for each value of $Z: \delta_{g}=f\left(z_{g}\right)=\delta_{z}$, and define $\mathbb{Z}$ as the set of values of $z_{g}$ present in the samples of siblings and switchers. The treatment effect estimated without FE using a sample of groups with $n_{g} \geq 2$, e.g. siblings, is:

$$
\hat{\delta}_{O L S}=\sum_{z \in \mathbb{Z}} \hat{\delta}_{z, O L S} \cdot \hat{\omega}_{z, O L S}
$$

where $\hat{\omega}_{z, O L S}$ is the sample analogue to $\omega_{z, O L S}$ :

$$
\omega_{z, O L S}=\frac{\left(\operatorname{Var}\left(D_{i} \mid n_{g} \geq 2, z_{g}=z\right) \cdot \operatorname{Pr}\left(z_{g}=z \mid n_{g} \geq 2\right)\right.}{\sum_{z^{\prime} \in \mathbb{Z}}\left(\operatorname{Var}\left(D_{i} \mid n_{g} \geq 2, z_{g}=z^{\prime}\right) \cdot \operatorname{Pr}\left(z_{g}=z^{\prime} \mid n_{g} \geq 2\right)\right.}
$$

and $\hat{\delta}_{z, O L S}$ is the OLS estimate without FE of the treatment effect for groups with $z_{g}=z$, and $\operatorname{Var}\left(D_{i} \mid n_{g} \geq 2, z_{g}=z\right)$ is the conditional variance of treatment for $n_{g} \geq 2$ and $z_{g}=z$.

The FE estimator for the same sample is:

$$
\hat{\delta}_{F E}=\sum_{z \in \mathbb{Z}} \hat{\delta}_{z, F E} \cdot \hat{\omega}_{z, F E}
$$

where $\hat{\omega}_{z, F E}$ is the sample analogue to $\omega_{z, F E}$ :

$$
\omega_{z, F E}=\frac{\left(\operatorname{Var}\left(D_{i} \mid F E, z_{g}=z, S_{g}=1\right) \cdot \operatorname{Pr}\left(z_{g}=z \mid S_{g}=1\right)\right.}{\sum_{z^{\prime} \in \mathbb{Z}}\left(\operatorname{Var}\left(D_{i} \mid F E, z_{g}=z^{\prime}, S_{g}=1\right) \cdot \operatorname{Pr}\left(z_{g}=z^{\prime} \mid S_{g}=1\right)\right.}
$$

and $\hat{\delta}_{z, F E}$ is the FE estimate of the treatment effect for groups with $z_{g}=z, \operatorname{Var}\left(D_{i} \mid F E, z_{g}=z\right)$ is the conditional variance of treatment for groups with $z_{g}=z$, net of family fixed effects.

Moving from OLS to FE, the $\hat{\delta}$ 's change and also the $\hat{\omega}$ 's change. The change in the $\hat{\delta}$ 's is how we usually interpret the move from OLS to FE: the change is from "between" (bad) variation to "within" (good) variation. But the full change also incorporates the different weightings of different values of $z_{g}$. If the OLS sample and the FE sample overlap in the covariates, we can decompose the difference between OLS and FE to identify how much is caused by the change in weights, $\hat{\omega}_{z}$, and how much is driven by the change in identification, $\hat{\delta}_{z}$, as:

$$
\begin{aligned}
\hat{\delta}_{F E}-\hat{\delta}_{O L S} & =\sum_{z \in \mathbb{Z}} \underbrace{\left(\hat{\omega}_{z, F E}-\hat{\omega}_{z, O L S}\right) \cdot\left(\alpha \cdot \hat{\delta}_{z, F E}+(1-\alpha) \cdot \hat{\delta}_{z, O L S}\right)}_{\text {Impact of } \Delta \text { weighting }} \\
& +\sum_{z \in \mathbb{Z}} \underbrace{\left(\hat{\delta}_{z, F E}-\hat{\delta}_{z, O L S}\right) \cdot\left(\alpha \cdot \hat{\omega}_{z, O L S}+(1-\alpha) \cdot \hat{\omega}_{z, F E}\right)}_{\text {OLS Bias }}
\end{aligned}
$$

with $\alpha \in[0,1]$ a researcher-determined weight. The impact of SI is captured in the first summation of Equation 6, which is a function of the disparity in regression weights $\hat{\omega}_{z}$ between OLS and FE, multiplied by an $\alpha$-weighted average of the $\hat{\delta}_{z, O L S}$ and $\hat{\delta}_{z, F E}$. Setting $\alpha=0$ in this term uses cross-section coefficients to assess the importance of changing the regression weights from 
OLS to FE. Setting $\alpha=1$ uses the FE coefficients to assess this. If there is important heterogeneity among both $w_{z}$ and $\delta_{z}$, these two extremes can provide useful benchmarks to compare against the OLS and FE estimates, as we do in Section 4.3. ${ }^{25}$

Equation 6 shows that SI matters more for the difference between OLS and FE when the gap in weights $\left(\hat{\omega}_{z, F E}-\hat{\omega}_{z, O L S}\right)$ is correlated with treatment effects $\hat{\delta}_{z}$ (across elements of $Z$ ), and there is heterogeneity in $\hat{\delta}_{z}$. This also raises the issue that the FE estimate may not be unbiased for the ATE for switchers, $E\left[\hat{\delta}_{F E}\right] \neq \sum_{z} \delta_{z} \cdot \operatorname{Pr}\left(z_{g}=z \mid S_{g}=1\right)$. When there are heterogeneous treatment effects, unbiasedness would require the weights $\omega_{z, F E}$ to match with the expected population shares across values of $Z$. This will typically not be the case, as shown theoretically in Chernozhukov et al. (2013); Gibbons, Suarez and Urbancic (2018); Imai and Kim (2019), and as we demonstrate in our empirical application below.

SI impacts the probability of each family size appearing in FE, in addition to potentially impacting the conditional variance of treatment. Existing methods to reweight FE estimates (Gibbons, Suarez and Urbancic, 2018; Imai and Kim, 2019) address only the conditional variance of treatment, and so can at best recover the ATE for switchers. Since switchers are typically not a population of interest, this raises concerns for the external validity of the FE estimator.

We use data from our empirical example to illustrate the change in the components of $\omega_{z}$ across OLS and FE. Panel A of Table 3 shows the proportion of the overall sample, the sibling sample, and the switcher sample comprised by families with $1,2,3,4$, or 5 or more kids. Moving from the sibling sample to the switchers sample, the share of the sample comprised of families with 5 or more kids roughly doubles (from $16.9 \%$ to $32 \%$ ), while the share of the sample comprised of 2-child families declines by $40 \%$ (from $34.5 \%$ to $21 \%$.)

Panel B of Table 3 shows the variance in Head Start across the same family sizes and samples, netting out group fixed effects for the switcher sample. Going from the siblings sample to the switcher sample, the variance approximately doubles for all family sizes. This suggests that the change in the conditional variance across OLS and FE plays a relatively minor role in shifting weights across groups in our setting. ${ }^{26}$

We then calculate $\hat{\omega}_{z, O L S}$ and $\hat{\omega}_{z, F E}$ in Panel C. Going from the sibling sample to the switchers sample, $\hat{\omega}_{2-\text { child }}$ declines by over $25 \%$ and $\hat{\omega}_{3 \text {-child }}$ declines by $15 \%$. Conversely, $\hat{\omega}_{5-\text { child }}$ nearly doubles from 0.134 to 0.243 , and $\hat{\omega}_{4-\text { child }}$ families increases by over $25 \%$.

\footnotetext{
${ }^{25}$ This decomposition is similar in form to Equation 13 in Loken, Mogstad and Wiswall (2012), which uses $\alpha=1 / 2$. However, we sum over a group-level covariate that is distinct from the treatment of interest, while Loken, Mogstad and Wiswall (2012) sum over values of an individual covariate (that varies within families), which is also the treatment of interest.

${ }^{26}$ We provide additional evidence that "undoing" the conditional variance weighting makes little difference in this application in Section 6.
} 


\section{Illustration of Consequences: Greater Returns to Head Start in Larger Families}

The treatment effect of Head Start also varies by family size in our applications. The first two columns of Panel A of Table 4 shows the estimated effects of Head Start on the likelihood of completing some college by the number of children in a family for our illustrative sample. We show the results with and without family fixed effects. In both specifications, the effect of Head Start is significantly higher among white children in families with 5 or more children and, once fixed effects are added, the effect of Head Start is monotonically increasing with the number of children in a family.

One possible explanation for this heterogeneity is that children with higher initial endowments receive greater parental investments in larger families, and also benefit more from Head Start (Aizer and Cunha, 2012). Another possibility is that Head Start substitutes for parental time, which is more scarce in larger families. Another interpretation is that this heterogeneity reflects the fact that other covariates correlated with family size, such as income, mediate the impacts of Head Start. This final explanation seems less likely, as we find that the heterogeneity in family size survives the inclusion of other interactions, as we discuss in Section 6.

The bottom of Panel A shows the number of Head Start switcher observations and effective observations in terms of cross-sectional and two-sibling switcher individuals. ${ }^{27}$ It shows that a total of 213 individuals are used to identify these coefficients, less than one tenth of the total sample, and that the variation is equivalent to 236 individuals in 2-person switching families. Hence, by including families with three or more children, on average, each observation is providing more variation than in a similar-sized sample of 2-child families. Further, the variation is equivalent to 731 individuals in a cross-sectional regression. This is because there is relatively little variation in Head Start in the full sample.

The larger Head Start effects we document for big families is not specific to the PSID. Columns (3) to (5) of Table 4 show the CNLSY FFE estimated effects of Head Start by family size for high school graduation, idleness, and having a learning disability. ${ }^{28}$ For each of these outcomes, the impact of Head Start for $5+$ child families is at least twice as large as the impact for 2 or 3 child families. For high school graduation, we also see a large impact for 4-child families, roughly double the impact for 2 and 3 child families. This implies that we should expect an increase in the coefficient going from OLS to fixed effects due to the change in weighting across the identifying samples, even without a change in the source of identification.

\footnotetext{
${ }^{27}$ For effective cross-sectional individuals, the denominator of Equation 3 is the variance of Head Start, residualized by the family mean of the covariates in the analysis For the effective number of two-person switcher individuals, the denominator is $\left[V\left(D_{i} \mid g\right) \cdot\left(n_{g}-1\right)\right] / n_{g}=\left[0.5^{2} \cdot(2-1)\right] / 2=0.125$.

${ }^{28}$ We focus on these outcomes because individuals that attended Head Start were found to fare significantly better on each of these outcomes in Deming (2009).
} 


\section{Extrapolating from Identifying to Target Population}

The difference between OLS and FE in the implicit weighting of heterogeneous treatment effects leads us to consider translating the FE estimates into an ATE for a (researcher-determined) population of interest. We propose a method to flexibly obtain the ATE for such populations of interest, which we refer to as "target" populations, and denote by an indicator $T_{g}$. To give a few examples, the target population could be a nationally representative sample, which is a common group of interest in applied work; or, more relevant for our application, families that are eligible for a means-tested program or families with at least one participant in a means-tested program.

\subsection{Assumptions and Proposition}

The reweighting method relies on four key assumptions, which are variants of those used for extrapolation from IV (Angrist and Fernandez-Val (2013); Aronow and Carnegie (2013)). First, we assume that Group ID conditional independence (Assumption 1, Equation 1) holds.

Second, we assume that conditional on observables, the true treatment effect is independent of a group's switching or target status. We refer to this as conditional fixed effect ignorability (CFEI). We use two propensity scores constructed from a vector of observable group characteristics, $\mathbf{X}_{\mathbf{g}}$, as the conditioning variables: $P_{x}:=\operatorname{Pr}\left[S_{g}=1 \mid \mathbf{X}_{\mathbf{g}}=\mathbf{x}\right]$ is the population propensity to be a switching group, and $Q_{x}:=\operatorname{Pr}\left[T_{g}=1 \mid \mathbf{X}_{\mathbf{g}}=\mathbf{x}\right]$ is the population propensity to be in the (researcher-determined) target group. ${ }^{29}$

\section{Assumption 2 (Conditional Fixed Effect Ignorability (CFEI)):}

$$
\begin{aligned}
& \mathbb{E}\left[Y_{i}(1)-Y_{i}(0) \mid S_{g}, P_{x}, Q_{x}\right]=\mathbb{E}\left[Y_{i}(1)-Y_{i}(0) \mid P_{x}, Q_{x}\right] \\
& \mathbb{E}\left[Y_{i}(1)-Y_{i}(0) \mid T_{g}, P_{x}, Q_{x}\right]=\mathbb{E}\left[Y_{i}(1)-Y_{i}(0) \mid P_{x}, Q_{x}\right]
\end{aligned}
$$

Assumption 2 is a strong assumption. CFEI eliminates, for example, a second type of Roy (1951)-type selection, whereby switchers have an unobserved quality that increases the effectiveness of treatment compared to observationally equivalent non-switchers. This assumption is required in order to extrapolate treatment effects from switchers to non-switchers. Analogous assumptions are made in the IV literature extrapolating from compliers to a target population (Angrist and Fernandez-Val, 2013; Aronow and Carnegie, 2013). The plausibility of Assumption 2 will depend on the context of the specific application.

In our application this assumption may be reasonable, because observables strongly predict both switching and target status. We have shown that the key determinants of $\operatorname{Pr}\left[S_{g}=1\right]$ are

\footnotetext{
${ }^{29}$ For instance, if the target group is "all individuals," $Q_{x}=1$ for each individual; while if the target group is "Head Start participants," $Q_{x}$ is the population probability that an individual in a group with $\mathbf{X}_{\mathbf{g}}=\mathbf{x}$ is a Head Start participant. Note that the target group is "all individuals," CFEI simplifies to the assumption that treatment effects are independent of switching status, conditional on the probability of being a switcher, $P_{x}$.
} 
family size and the underlying probability of Head Start participation. Family size is observable, and observable covariates, such as family income, can take us a long way in predicting program participation. Likewise, the family-level determinants of $\operatorname{Pr}\left[T_{g}=1\right]$ for a target such as Head Start participants will be largely tied to observable eligibility requirements for the program, such as income and household size, which together determine the income-to-poverty ratio.

However, there may be unobservables that determine switching or target status, and which are also correlated with treatment effects. To address this concern, we develop two testable implications of Assumption 2 at the end of this subsection.

In contexts where this assumption may be too strong, the reweighting procedure can still help to reduce bias, since balancing on observables will likely reduce the degree of bias that is driven by mismatch on unobservables. Andrews and Oster (2019) develop this idea formally in the context of extrapolating from an experiment to a larger target population. This improvement relies on the assumption that the direction of bias from selection on observables is the same as the direction of bias from selection on unobservables, which may be plausible in many cases. ${ }^{30}$

\section{Assumption 3 (Correct Propensity Score Specification):}

$$
\begin{aligned}
& \operatorname{Pr}\left(S_{g}=1 \mid \mathbf{X}_{\mathbf{g}}\right)=F\left(\theta ; \mathbf{X}_{\mathbf{g}}\right) \\
& \operatorname{Pr}\left(T_{g}=1 \mid \mathbf{X}_{\mathbf{g}}\right)=G\left(\chi ; \mathbf{X}_{\mathbf{g}}\right)
\end{aligned}
$$

Third, we assume that the propensity scores that we estimate have the correct functional form, with $F(\cdot)$ and $G(\cdot)$ known, and $\theta$ and $\chi$ parameters to be estimated. In our application, we model $F(\cdot)$ and $G(\cdot)$ jointly as a multinomial logit.

\section{Assumption 4 (Overlap):}

$$
\text { If } Q_{x}>0 \text {, then } P_{x}>0
$$

Fourth, we require a positive probability of being a switcher for each value of $Q_{x}$ in the target group, which ensures that we can use the switcher sample to recover the distribution of treatment effects in the target sample. Since some covariate values may not be observed in the switcher sample, this assumption implicitly places some restrictions on the relationship between treatment effects and these covariates. For example, since we do not observe 1-unit groups ("singletons") in the switcher sample but they are present in some of our application target populations, ${ }^{31}$ we cannot allow treatment effects for singletons to be outside the support of the treatment effects of switchers. This precludes us from including an indicator for singletons in $\mathbf{X}_{\mathbf{g}}$. In our application

\footnotetext{
${ }^{30}$ For example, we show that larger families are likely to have more variation and larger treatment effects. Such families may also be more motivated (or better equipped) to seek out information about public programs, which would also predict greater variation and larger treatment effects.

${ }^{31}$ Singletons comprise $6 \%$ of Head Start participants in the CNLSY, and $18 \%$ of Head Start participants in the PSID.
} 
we preserve overlap by assuming that treatment effects are the same for all groups with 2 or fewer units, which allows us to extrapolate treatment effects for singletons from 2-unit groups. ${ }^{32}$ An alternative approach is to extrapolate treatment effects for never-switchers using functional form assumptions, which we discuss under extensions below.

Proposition 1. Define the re-weighted FE estimator for target population $t$ as

$$
\widehat{\delta^{t}}:=\frac{1}{\sum_{i} \mathbf{1}\left(S_{g(i)}=1\right)} \sum_{i \mid S_{g(i)}=1} \widehat{w_{g(i)}^{t}} \cdot \hat{\delta}_{g, F E},
$$

with $\widehat{w_{g(i)}^{t}}$ our estimate of $w_{g(i)}^{t}$,

$$
w_{g(i)}^{t}:=\frac{Q_{x} \cdot \operatorname{Pr}[S=1]}{P_{x} \cdot \operatorname{Pr}[T=1]}
$$

Under Assumptions 1 through $4, \widehat{\delta}^{t}$ is consistent for the ATE of the target population, $\mathbb{E}\left[Y(1)-Y(0) \mid T_{g}=1\right]$.

The proof is in Appendix A. Intuitively, the weights are increasing in $Q_{x}$ and decreasing in $P_{x}$, such that we upweight observations that are more similar to the target, and downweight observations that are overrepresented in the switching population. The other terms in the weight, $\operatorname{Pr}[S=1]$ and $\operatorname{Pr}[T=1]$, are constant across groups. The treatment estimate for each switcher group $g$ is weighted proportionately to match the share of the target population with observable characteristics matching $g$, which gives the ATE under the assumptions above. ${ }^{33}$ Because the weights increase as $\widehat{P_{x}}$ gets close to 0 , for implementation we suggest directly examining the estimated weights, and using caution if there are values of $\widehat{P_{x}}$ that are extremely small.

\section{Testable Implications}

CFEI requires that treatment effects should be balanced across $T_{g}$, conditional on $P_{x}$ and $Q_{x}$ (Equation 8). Hence, switchers in the target population should on average have the same treatment effects as switchers in the non-target population once observables are balanced across the two groups. This is potentially testable if some switchers are not in the target population (i.e. if the target is not "everyone," "multi-unit groups," or otherwise contains the set of switchers $G_{S}$.) For instance, if the target population is families that participate in a safety net program, groups that live in rural areas, or firms that are in a particular industry, we could test whether treatment effects are different for participants and non-participants, rural and urban individuals, etc.

CFEI also requires that treatment effects should not vary across switchers and non-switchers (Equation 7). This could be violated, for example, if families with a lower return to Head Start

\footnotetext{
${ }^{32}$ We implement this by including an indicator for " 1 or 2 child families" in $\mathbf{X}_{\mathbf{g}}$ together with indicators for other family sizes. Alternatively, the target group can be defined to only include families that are ever switchers, such as "siblings" or "multi-child Head Start families."

${ }^{33}$ See Appendix A for a simple derivation of the weights.
} 
make less of an effort to have any of their children attend, or if families with a higher return to Head Start make a greater effort to have all of their children attend. Because we never observe the treatment effects of non-switchers, we can not directly test for this type of violation. However, this type of violation generates a testable implication: if families systematically choose the number of children to attend Head Start based on private information, we should also expect to see this pattern within switchers. In particular, if switchers act on private information about the size of treatment effects, then switcher families that have a higher fraction of children that attend Head Start should experience systematically larger gains. Thus, a useful diagnostic test is whether treatment effects vary with the number or fraction of treated units, holding constant the size of the family. ${ }^{34}$

We implement both of these tests for our setting in Section 6.2.1.

\subsection{Reweighting Methodology}

In order to implement this reweighting strategy, we first need to obtain estimates of $P_{x}$ and $Q_{x}$. These two elements can be calculated using a multinomial logit model where the outcomes are the four possible combinations of having $S_{g}=1$ or $S_{g}=0$, and $T_{g}=1$ or $T_{g}=0$. $\hat{Q_{x}}$ is then constructed as the sum of the predicted $\operatorname{Pr}\left(T_{g}=1, S_{g}=0\right)$ and the predicted $\operatorname{Pr}\left(T_{g}=1, S_{g}=1\right)$ for each group. $\hat{P}_{x}$ is constructed as the sum of the predicted $\operatorname{Pr}\left(T_{g}=1, S_{g}=1\right)$ and the predicted $\operatorname{Pr}\left(T_{g}=0, S_{g}=1\right)$ for each group.

With these weights in place, the ATE for the target population can be estimated in one of two ways. The first is a two-step "post-regression weighting" of $\hat{\delta_{g}}$, where $\hat{\delta_{g}}$ is estimated from a regression of the outcome on interactions between $D_{i}$ and group-specific dummies. Aggregating $\widehat{w_{g(i)}^{t}}$ to the group-level and performing a normalization, we obtain the two-step estimation weights, $\hat{s_{g}^{t}}=\frac{\widehat{w_{g(i)}^{t}} \cdot n_{g}}{\sum_{g^{\prime} \in G_{s}} \widehat{w_{g(i)^{\prime}}^{t}} \cdot n_{g^{\prime}}}=\frac{\frac{\hat{Q}_{x}}{P_{x}} \cdot n_{g}}{\sum_{g^{\prime} \in G_{S}} \frac{\hat{Q}_{x}}{P_{x}} \cdot n_{g^{\prime}}}$. The 2-step ATE is then:

$$
\widehat{\delta_{2 s t e p}^{t}}=\sum_{g \in G_{S}} \hat{s_{g}^{t}} \cdot \hat{\delta_{g}}
$$

A second approach is to obtain the ATE in a single step using "in-regression weights." For this, we need to incorporate an additional element to our p-score weights that "undoes" the conditional variance weighting of FE: $v_{g}=\left(\operatorname{Var}\left(D_{i} \mid g(i)=g\right)^{-1}\right.$ (Gibbons, Suarez and Urbancic, 2018). ${ }^{35}$ The ATE can then be estimated from a one-step regression using $\widehat{w_{g(i)}^{t}} \cdot v_{g}$ as regression weights. ${ }^{36}$

Our results in section 4.1 assume simple random sampling. In our application, we adjust our reweighting procedure to incorporate survey weights. First, we weight our multinomial logit using

\footnotetext{
${ }^{34}$ Controlling for group size allows us to distinguish whether families have a higher fraction attend due to selection or due to other factors, such as the mechanical relationship between family size and probability of being a switching family, or unmeasured variables that are correlated with family size.

${ }^{35}$ This variance is computed using the population formula, (dividing by $n_{g}$ ), rather than the sample formula (dividing by $n_{g}-1$ ).

${ }^{36}$ In the special case where the target group is "switchers," then $P_{x}=Q_{x}, \widehat{w_{g(i)}^{t}}=1$, and the one-step weight simplifies to $v_{g}$, so that we recover the ATE for switchers.
} 
survey weights. The resulting $\widehat{w^{t}}$ then has the interpretation of translating between "population switchers" and "population target observations." Second, we multiply $\widehat{w_{g(i)}^{t}}$ by survey weights for both the one- and two-step reweighting procedures. We obtain standard errors for all estimates by bootstrapping.

\subsection{Special Case: Univariate Heterogeneity}

If the source of heterogeneity in estimates is a single, discrete covariate, we can obtain further insight from performing the decomposition captured in Equation 6. Taking the OLS family-sizespecific coefficients from column (1) of Table 4 and reweighting by the fixed-effects regression weights ( $\alpha=0$ in Eq. 6 ), we obtain a weighted coefficient of 0.069 , shown in the bottom row of Table 4. This implies that approximately $1 / 3$ of the change from OLS to FE $\left(\frac{0.069-0.049}{0.12-0.049}\right)$ is driven by the change in weighting across family sizes; with the other $2 / 3$ driven by change in identifying variation. Further, reweighting the FE estimates using the OLS weights ( $\alpha=1$ in Eq. 6) produces a coefficient is 0.083 . This implies that the imbalance in family size alone causes the FFE estimate to be $50 \%$ higher than the estimates without FE.

\subsection{Monte Carlo Experiments}

We perform a Monte Carlo analysis to examine the properties of our proposed reweighting estimators. We use naturally occuring selection into identification from our PSID application and model treatment effects for three settings, allowing the true ATE to be known. Each setting has a different model of heterogeneity in treatment (assumed to be known to the researcher), which determines the covariates that we use to generate the propensity score.

We generate the data for the Monte Carlo as follows. We construct an untreated outcome for each individual by running a linear probability model of attainment of "some college or more" on demographic variables, income during childhood, and parental education. We then use the estimates from this model to generate a prediction of a continuous probability that an individual completes some college, $X_{i g} \cdot{ }^{37}$ All simulations start with this constructed variable $X_{i g}$ and the

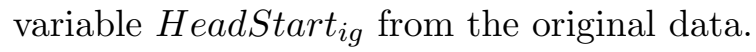

We then construct latent outcomes inclusive of treatment as $Y_{i g}^{*}=X_{i g}+\beta_{i g} H_{e a d S t a r t}$, where $\beta_{i g}$ is the treatment effect of Head Start and varies across different models of heterogeneity. We always scale $Y_{i g}^{*}$ to ensure that these probabilities lie within the range $[0,1]$. We then randomly generate the binary outcome variable as $\operatorname{Pr}\left(Y_{i g}=1\right)=Y_{i g}^{*}$.

We consider three models of heterogeneity in treatment effects. First, $\beta_{i g}=0.08$. We use the variable $X_{i g}$ to generate propensity scores. Second, $\beta_{i g}=0.192$ for large families (with 4 or more siblings) and $\beta_{i g}=0$ for small families ( 3 or fewer children). We use a dummy variable for "large family" to generate propensity scores. Third, we allow the treatment effect heterogeneity

\footnotetext{
${ }^{37}$ For simplicity, we restrict the sample to those with $X_{i g} \in[0,1]$ at baseline.
} 
to vary smoothly: $\beta_{i g}=0.08 \cdot\left(1-\frac{X_{i g}-\overline{X_{i g}}}{s . d .\left(X_{i g}\right)}\right) \cdot \frac{1}{3}$, with $\overline{X_{i g}}$ and $s . d .\left(X_{i g}\right)$ the sample mean and standard deviation of $X_{i g}$. This produces a treatment effect that is larger for lower-baselineprobability individuals and ranges from 0.01 to 0.15 for most of the population. For this more complex treatment effect, we generate propensity scores in two ways: using $X_{i g}$ and, more flexibly, using a spline in $X_{i g}$, with knots at the $5^{t h}, 20^{t h}, 50^{t h}, 80^{t h}$, and $95^{t h}$ percentiles of $X_{i g}$. The latter model presumes that the researcher has some intuition that the treatment effect or selection into identification may vary non-linearly with baseline outcomes.

We run 3,000 replications of our Monte Carlo simulation. In each replication, we keep track of the true ATE for each target population of interest, the FE estimate of the treatment effect, and the reweighted regression estimate of the treatment effect for each target population. ${ }^{38}$ The FE estimate is the same for all target populations. We consider four target populations: (i) individuals in Head Start switching families; ${ }^{39}$ (ii) all siblings; (iii) all individuals in the sample (including singletons); and (iv) all Head Start participants. We multiply all estimates by 1,000 for easier readability.

Panel A of Table 5 presents results for the model with constant treatment effects. In this setting, the average treatment effect is the same for all target populations, all estimators are unbiased, and the FE model is the minimum variance estimator. The reweighting estimators have mean squared errors 3 to $20 \%$ larger than for OLS.

Panel B of Table 5 presents results for the model with zero treatment effect for small families, and large treatment effects for large $(4+$ children) families. It shows that for every target population, FE is biased, while the reweighting estimator is always unbiased. This improvement in bias over FE leads to much better mean squared error results for the reweighting estimator. ${ }^{40}$

Panels $\mathrm{C}$ and D of Table 5 examine the third model with heterogeneous treatment effect that varies with $X_{i g}$. Here the FE model has relatively little bias for the switcher and Head Start participant targets (-0.2 p.p, and -0.08 p.p. on a base of 9 p.p.), but has much larger bias for the remaining targets. Panel $\mathrm{C}$ shows that the regression reweighting estimator which uses $X_{i g}$ in the propensity score estimation has less bias than FE for all target populations, with no detectable bias for the switcher, or Head Start populations. The small bias for the reweighting estimator for the other target populations results from an imperfect balance in the $X_{i g}$ variable, even after reweighting. ${ }^{41}$

Panel D shows that when we re-estimate the model including a spline in $X_{i g}$ in the propensity score estimation, the reweighting estimator has no detectable bias for any of the target groups. This

\footnotetext{
${ }^{38}$ Both post-regression and in-regression reweighting produce the same results.

${ }^{39}$ This will not necessarily be the same as the FE estimate because of differences in the conditional variance across families.

${ }^{40}$ In results not reported, we have examined adding $X_{i g}$ as a covariate to the propensity score estimation stage in this model. This introduces a small amount of bias in the reweighting estimator (-0.1 p.p., relative to the 2 to 3 p.p. bias in FE) for the "siblings" and "all" target groups.

${ }^{41}$ This is because $\operatorname{Pr}\left(S_{g}=1\right)$ is misspecified as a linear function of $X_{i g}$, which causes us to misassign the weight for each treatment effect.
} 
suggests that allowing for greater flexibility in the functional form relationship between covariates and the propensity score can achieve greater reductions in bias.

Overall, the results of this exercise show that that the reweighted estimator has significantly less bias than FE for the types of treatment effect heterogeneity we consider, and can be successfully targeted toward different target populations. Consistent with the conditioning on observables requirements of this estimator, its performance is best when it is given the appropriate covariates for the particular type of heterogeneity, and when the model for the probability of switching is correctly specified.

\section{Extensions}

Projecting Treatment Effects for "Never-Switchers" As noted above, the reweighting estimator in Proposition 1 only recovers the ATE for the target population if (i) the target does not include never-switchers or (ii) if the treatment effects for never-switchers in the target can be assumed to be the same as some other target groups with $P_{x}>0$. Otherwise, the reweighting estimator only obtains the ATE for the subset of the target with $P_{x}>0$, for whom treatment effects are identified.

A slight variant of (ii) that could also enable the recovery of the target ATE is to extrapolate treatment effects for never-switchers. This requires a stronger form of CFEI: that treatment effects are not only a function of observable characteristics, but that the researcher can correctly specify the functional form of this relationship. ${ }^{42}$ The weighted average of estimated treatment effects for $P_{x}>0$ and extrapolated effects for $P_{x}=0$ would then give the ATE for the target group.

Unit $i$ Covariates Thus far, we have ignored covariates in our models. However, researchers may want to include individual-level covariates $C_{i}$ ( that vary across $i$ units within a group) in their models in order to (i) make Assumption 1 more reasonable; (ii) improve precision of estimates (iii) allow extrapolation to target groups defined at the unit level. Once these covariates are included, the typical intuition that "groups with variation in treatment" provide identification breaks down. This is because for some groups, who we refer to as "residual switchers," there can be variation in the treatment residualized of $C_{i}$, even if there is no within-group variation in $D_{i} \cdot{ }^{43}$ Thus, treatment effects can also be estimated for residual switchers; however, identifying variation comes from from within-family variation in $C_{i}$, not $D_{i}$. In Appendix A.3 we discuss additional considerations related to residual switchers, including how one can quantify the contribution of residual switchers to estimates, and how our key assumptions and proposition can be extended to accomodate $C_{i}$.

Re-weighting misspecified FE models We have also focused on cases where Assumption 1 (CIA) holds, and the within-group comparisons form unbiased estimates of group-level treatment

\footnotetext{
${ }^{42}$ See Appendix A.1.1 for a formalization of this assumption and an extension of Proposition 1 using exrapolation.

${ }^{43}$ See Appendix A.3 for a formalization of this.
} 
effects. If instead there are violations of Assumption 1, then reweighting estimates can affect the resulting bias. The impact of reweighting on the misspecification bias will depend on the covariance (across groups) of group-specific bias, bias $s_{g}$, and the impact of the reweighting procedure on the weight given to each group: $E_{g}\left[\left(w_{g}^{t}-\omega_{g, F E}\right) \cdot b i a s_{g}\right]$. For example, suppose that smaller-sized groups had a more positive bias and that the reweighting increased the weight of these groups. This would make the misspecification bias more positive, and in principle could possibly lead to a net increase in the overall bias. This suggests that researchers may want to consider violations of CIA by group characteristcs (e.g., group size) before reweighting.

Nonlinear Functional Form Next, we relax the linear functional form assumption. One reason this may make a difference is that conditional or fixed effect logit models use only "double switchers," families with variation in both the outcome variable and the treatment variable. In Appendix E, we show that the biases from SI are similar in the linear probability model and conditional logit, and that the reweighting we propose is equally effective at reducing bias in both cases.

Continuous $D_{i} \quad$ Finally, while we have focused on the case where $D_{i}$ is binary, it is worth noting that SI can also be present when $D_{i}$ is continuous (since $\hat{\delta}_{g, F E}$ is still only estimated for switching families.) It is not clear how frequently this will manifest in practice, however, since groups are more likely to have variation in a continuous covariate. Even so, it may still be worthwhile to verify the number of switchers, since there may be persistent bunching at one value of $D_{i}$, such as at zero maternal income or at zero instances of an uncommon event.

\section{Effects of Head Start}

\subsection{Data and Replication of GTC and Deming (2009)}

We now turn to examining the impact of Head Start on long run outcomes using the PSID and CNLSY, which were used to analyze this question in GTC and Deming (2009).

\subsubsection{PSID}

The PSID sample includes the sample of individuals surveyed in the PSID by 2011. The PSID began in 1968 as a survey of roughly 5,000 households and has followed the members of these founding households and their children longitudinally. The longitudinal nature of the study allows sibling comparisons during early adulthood as well as later in life.

We begin our analysis with a replication of GTC. The sample includes all black or white individuals born between 1966 and 1977, and excludes Hispanic individuals.We provide a detailed description of our replication of GTC in Appendix D. Despite some minor differences, the two PSID samples are qualitatively similar. The summary statistics are often within a third of a standard

deviation of each other. Moreover, the estimated effects of Head Start in this sample are similar 
to those estimated in GTC. We find large (23 p.p.) and significant effects of Head Start on the probability that whites attain some college, and large point estimates (9.3 p.p.) for high school graduation, though in our case these are not statistically significant. We do not find that Head Start meaningfully reduces the probability of committing a crime. ${ }^{44}$

For the remaining analyses from here, we use a sample that substantially expands and modifies the GTC sample. First, we expand the sample to include individuals born between 1978 and 1987. The individuals in these cohorts were too young when the analysis in GTC was performed to observe their education and early career outcomes. Second, we include older siblings of all individuals, including those born prior to 1966. These early cohorts were typically too old to benefit from the introduction of Head Start, and serve as a plausible control group for the early cohorts.

In addition to modifications of the sample, we also expand the number of outcomes under analysis in order to gain a more extensive understanding of the channels by which Head Start affects children's lives. We follow the established practice of distilling the measures to summary indices to lessen problems with multiple hypothesis testing (see, e.g., Anderson, 2008; Kling, Liebman and Katz, 2007; Hoynes, Schanzenbach and Almond, 2016). We create four indices to capture economic and health outcomes observed for individuals at age 30 and 40. The "economic sufficiency index" includes measures of educational attainment, receipt of AFDC/TANF, food stamps, mean earnings, mean family income relative to the poverty threshold, the fraction of years with positive earnings, the fraction of years that the individual did not report an unemployment spell, and homeownership. The "good health index" summarizes the following component measures: non-smoking, report of good health, and negative of mean BMI. ${ }^{45}$

The process of creating each index follows the procedure described in Kling, Liebman and Katz (2007). In particular, we standardize each component of the index by subtracting the mean outcome for non-treated children, defined as children that did not attend any form of preschool, and then dividing the result by the standard deviation of the outcome for non-treated children. The summary index takes a mean of these standardized measures. ${ }^{46}$ We also extract the first principal component of the standardized variables for "economic sufficiency" and for "good health". Later we use these as alternative outcome variables.

Appendix Table B.3 reports sample descriptive statistics for the expanded sample we construct. For ease of comparison with our earlier replication, we include means for the entire sample, the subsamples of Head Start participants/non-participants, and for the sample of individuals with

\footnotetext{
${ }^{44}$ In some subsamples, we even find an effect in the opposite direction. We believe these cases are driven by situations where there are rather few observations identifying the coefficients, and that the lack of correspondence may be driven by very minor (and un-diagnosable) differences in specification and/or dataset construction.

${ }^{45}$ See Appendix Table B.5 for descriptive statistics of the inputs to the indices.

${ }^{46}$ Consistent with Kling, Liebman and Katz (2007), we generate a summary index for any individual for whom we observe a response for one component of the index. Missing components of the index are imputed as the mean of the outcome conditional on treatment status. For example, if a former Head Start participant is missing an outcome, it is imputed as the mean outcome of other Head Start participants. Likewise for other preschool, or non-preschool participants.
} 
siblings. We present the means of the analyzed outcomes in Appendix Table B.4. ${ }^{47}$

\subsubsection{CNLSY}

We obtain the CNLSY sample from the Deming (2009) replication files, which ensures that the samples are identical. The CNLSY is a longitudinal survey that follows the children born to the roughly 6,000 women that took part in the NLSY79 survey. The sample we use includes all children who were at least 4 years old by 1990 .

\subsection{Head Start Estimation}

The empirical strategy takes advantage of within-family variation in participation in Head Start to identify the long term impact of the program. Following GTC and Deming (2009), we estimate:

$$
Y_{i g}=\alpha+\beta_{1} \text { HeadStart }_{i g}+\beta_{2} \text { OtherPreSchool }_{i g}+\mathbf{X}_{\mathbf{i g}} \gamma+\delta_{g}+\varepsilon_{i g}
$$

where $Y_{i g}$ represents a long-term outcome for individual $i$ with mother $g$. HeadStart ${ }_{i g}$ indicates whether a child reports participation in the program, and Other PreSchool ${ }_{i g}$ indicates participation in other preschool (and no participation in Head Start). These two variables are in this way defined so as to be mutually exclusive, with "neither Head Start nor other preschool" as the omitted category. ${ }^{48} \delta_{g}$ is a mother fixed effect which enables comparisons across siblings with a shared mother. The vector $\mathbf{X}_{\mathbf{i g}}$ includes a large number of controls for individual and family characteristics to absorb differences in personal and household characteristics which may be correlated with one's participation in Head Start and long term outcomes. These controls vary due to data availability across sources and specification used in earlier work, but fall into three broad categories: demographics, family background, and family economic circumstances during early childhood. ${ }^{49}$

Missing control variables are imputed at the mean, and we include an indicator variable for these imputed observations. We cluster standard errors on mother id, and use population-representative weights where appropriate. ${ }^{50}$ When $Y_{i g}$ is binary, we estimate linear probability models as a main specification and check the sensitivity of our results to alternative models.

\footnotetext{
${ }^{47}$ Appendix Table B.5 includes summary statistics for the inputs to the summary indices. Appendix Tables B.6, B.7, and B.8 contain the number of observations for each outcome and control variable in the analysis .

${ }^{48}$ Since Head Start only became available in 1965, we recode Head Start attendance to be "other preschool" for the 1961 and older cohorts.

${ }^{49}$ For the PSID, these include: individual's year of birth, sex, race, and an indicator for being low birth weight, mother and father's years of education, an indicator for having a single mother at age 4, 4-knot splines in annual family income for each age 0,1 , and 2 , a fourth spline based on average family income between ages 3 and 6 , indicators for mother's employment status at ages 0,1 , and 2, and household size at age 4 . For the CNLSY, these include: health conditions before age 5, PPVT test score at age 3, measures of birth weight, measures of mother's health and health behaviors, mother's working behavior and income prior to age 4, indicator for being first born, participation in Medicaid, relative care, and indicators for early care types.

${ }^{50}$ We follow our predecessors' weighting practices: for the PSID, we generate representative population weights from the 1995 March CPS, and for the CNLSY do not use weights.
} 
The coefficient of interest is $\beta_{1}$, the impact of Head Start on long term outcomes compared to no preschool. We generate propensity score weights to obtain the ATE for three target populations: (1) Head-Start-eligible individuals, based on family income between ages 2 and $5 ;^{51}$ (2) all Head Start participants; and (3) all siblings. For parsimony, we use a subset of the variables in Table 2 to generate the propensity score for each race: year of birth, gender, mother's years of education, income at age 3 , and income at age 4, and indicators for family size (grouping together 1 and 2 child families). ${ }^{52}$ We include results for the post-regression weighting method; results are qualitatively similar when we use in-regression weighting.

\subsubsection{Evidence on Model Assumptions: Identifying and CFEI}

The standard test of the identifying assumption (Assumption 1) is to look for balance in observables across siblings within families. Deming (2009) finds little evidence that Head Start attendance is correlated with observable differences across siblings, which suggests that the magnitude of selection may be small. In Appendix Table B.9, we examine the plausibility of the identifying assumption in the PSID by testing the correlation between participation in Head Start and observable pre-Head Start individual and family characteristics (and omitting these characteristics as controls where relevant). For the white sample which forms our focus, there are few statistically significant correlations, which suggests that the assumption may be reasonable. ${ }^{53}$

As a first test of CFEI (Assumption 2), Appendix Table B.10 analyzes whether treatment effects vary across observationally comparable families in the target and non-target populations. Recall that CFEI implies that conditional on $P_{X}$ and $Q_{x}$, treatment effects should be independent of observable characteristics, including membership in the target population. To implement this test, we regress estimated family-specific treatment effects on an indicator for whether an individual is a member of the target population, employing traditional inverse propensity score weights to balance observables (and therefore treatment effects, if CFEI holds) between target and non-target switchers. ${ }^{54}$ When target status varies within family, another way of viewing this test is as asking: is the treatment effect related to the share of individuals in the family that are in the target? This test passes with no sign of systematic differences across target and non-target individuals across all outcomes, two datasets, and two different target populations (Head-Start-eligible and Head-Start-participants), although the standard errors are often large. ${ }^{55}$

\footnotetext{
${ }^{51} \mathrm{An}$ individual is considered Head-Start-eligible if at any point between the ages of 2 and 5 her family income was below $150 \%$ of the poverty level, to account for our imperfect ability to observe reportable income.

${ }^{52}$ Results are similar when we substitute family size indicators with linear and quadratic terms in family size.

${ }^{53}$ For the black sample, participation in Head Start is correlated with a greater likelihood of having higher income at age 1, and lower income at age 2, which may raise concerns that black families may tend to send their children to Head Start after a rupture in the family or after an income shock. However, given the many hypotheses being tested in this table, these significant findings might be spurious; and these results are somewhat sensitive, becoming insignificant when we drop observations with imputed controls.

${ }^{54}$ For target individuals the weights are $1 / \widehat{\operatorname{Pr}}\left[T_{i}=1, S_{g}=1 \mid X_{i g}\right]$, and for non-target individuals the weights are $1 / \widehat{\operatorname{Pr}}\left[T_{i}=0, S_{g}=1 \mid X_{i g}\right]$.

${ }^{55}$ We have run analogous models at the family level, which give qualitatively similar results.
} 
As a second, related, test, we examine whether the impact of Head Start varies with the fraction of children that attend Head Start in a family (which could signal that switchers have private information about the gains from Head Start). Appendix Tables B.11 and B.12 show the results for the PSID and CNLSY samples, respectively. In the PSID, we find no significant interaction with the fraction of children that attend Head Start. In the CNLSY, we find that 5-child families that have more children attend Head Start have smaller improvements in high school and larger improvements in learning disabilities, although this relationship appears to be driven by a very small number of individuals. Averaging across all family sizes, however, we find no significant relationship between the fraction that attend Head Start and treatment effects. This lends further support to our assumption that it is appropriate to extrapolate from switchers to non-switchers. ${ }^{56}$

Finally, extrapolation from switchers to the target population also requires that switchers have appropriate representation over the distribution of $P_{x}$ and $Q_{x}$. Assumption 4 (Overlap) implies that this will hold asymptotically, but for a finite sample this should be directly checked. Thus, we compute the fraction of target observations that lie within the convex hull of $P_{x}$ and $Q_{x}$ for the switcher observations. ${ }^{57}$ Appendix Figure B.4 shows this graphically for the "Head Start participant" target. In total, $95 \%$ of Head Start participants lie within the convex hull of the switcher sample. Of those that are outside, most are close to the convex hull. We interpret this magnitude of violation of the overlap assumption as mild enough to disregard in our subsequent analysis. ${ }^{58}$

\subsection{Head Start Results}

\subsubsection{Reweighted Estimates}

We begin by presenting results for our illustrative outcome, attainment of some college for whites in the PSID, in Panel A of Table 6. Column (1) of the table presents the estimated impact of Head Start on some college in GTC, column (2) presents the results using our expanded sample, and columns (3) to (5) present reweighted estimates for the three target populations. As reported earlier, we estimate that Head Start increases the likelihood of attaining some college by a statistically significant 12 p.p. (se: 0.053 ) using the baseline FFE model. This estimate is $57 \%$ smaller than the estimate reported in GTC, 0.281 (se: 0.108). ${ }^{59}$ The standard errors are also roughly 50\% smaller,

\footnotetext{
${ }^{56}$ If we take the negative slope on the fraction attending at face value (and disregard the noise in the estimates), it suggests that for the target population of HS participants, our treatment effects might be too large. In contrast, for a target population that has many non-switcher non-participants, our estimated treatment effect might be too small. If there are systematic differences, one approach to correct for this would be to include fraction participating as an additional covariate, with dummies that span bins that cover both switching and non-switching families; although in that case, it may also be prudent to place less emphasis on the reweighted estimates.

${ }^{57}$ Traditional methods based on a single propensity score involve checking the overlap of the propensity score distributions. We extend this idea to two dimensions.

${ }^{58}$ Appendix Figure B.5 shows an analogous graph for the Head Start eligible sample. Here, $90 \%$ of target individuals are within the convex hull, and all of those outside are very close to it.

${ }^{59}$ We show in the appendix that this discrepancy is not due to faulty replication of the GTC estimates in a smaller sample. We estimate a coefficient of 0.232 (se: 0.094) for this sample and outcome in our replication.
} 
corresponding to the roughly tripling of sample size $(2,986$ compared with 1,036$)$.

As we foreshadowed earlier, these estimates are unlikely to represent the ATE for policy relevant populations, such as the Head Start eligible population and Head Start participants. Figure 3 shows a scatter of the FFE weights and the Head-Start-representative weights for each family in the white sample, divided by 2- to 3-child families (Panel A) and 4-or-more-child families (Panel B). The larger (smaller) markers signify that the estimated effect of Head Start on some college for the family is above (below) median. We also include a 45 degree line for reference. The figure shows that, in general, the Head-Start-representative weights are higher than the FFE weights for small families that experience smaller impacts of Head Start. Conversely, the representative weights are lower relative to the FFE weights for large families that experience larger impacts of Head Start. Hence, we should expect the reweighted estimates to show a reduced impact of Head Start relative to FFE. This figure also shows that there are no weights that are unduly large, so we do not trim any observations based on $\widehat{P_{x}}$.

The reweighted estimates of the impact of Head Start for the eligible, participant, and sibling populations are 0.052, 0.021, and 0.064, respectively, and are all statistically insignificant. ${ }^{60}$ Setting aside the lack of precision in the estimates, these represent moderately large impacts relative to the 43.7\% average rate of college going among Head Start eligible children. But relative to the FFE coefficient, these effects imply a $46 \%$ to $83 \%$ smaller impact on college attendance. Putting these estimates in broader perspective, they are 45 to $91 \%$ smaller than the unadjusted estimates for all participants from other FFE studies (Bauer and Schanzenbach, 2016; Deming, 2009) and 51\% smaller than the estimate from the county roll-out of Head Start (Bailey, Sun and Timpe, 2018), although the lower end of the confidence intervals for these estimates include our ATE.

Panel B of Table 6 presents results for the Economic Sufficiency Index in the PSID. Our FFE estimate shows a statistically insignificant 0.023 SD decline in this index associated with Head Start. When we reweight the effects, we find slightly larger negative effects for Head Start eligible children and Head Start participants, and a positive effect (0.02 SD) for siblings. It bears emphasizing, though, that the results are not precisely estimated, such that the $95 \%$ confidence intervals allow for a sizeable positive impact of Head Start in spite of the small or negative point estimate. For example, the confidence interval allows for a Head-Start-induced improvement of $0.15 \mathrm{SD}$ or a reduction of $0.23 \mathrm{SD}$ for Head Start participants. This limits our ability to make firm conclusions about Head Start's impact on this outcome.

The following four panels of Table 6 show the CNLSY FFE estimates, those reported in Deming (2009) and our replication, and our reweighted estimates. The panels report effects for high school graduation, idleness (not in school or at work), diagnosis of a learning disability, and poor health (based on self-reported health status). The FFE estimates indicate that Head Start leads to an 8.5 p.p. increase in high school graduation $(p<0.01)$, a 7.2 p.p. decline in idleness $(p<0.10)$, a

\footnotetext{
${ }^{60}$ This reflects both the reweighting of heterogeneous effects across family sizes that we documented earlier, as well as slight changes to the family-size specific treatment effects (due to changes in the weighting of families that are less-represented along other covariates within a family size).
} 
5.9 p.p. decline in having a learning disability $(p<0.01)$, and a 6.9 p.p. decline in reporting poor health $(p<0.01)$. The reweighted estimate for participants for high school is $44 \%$ smaller, and not statistically significant. We also see substantial $24 \%$ and $28 \%$ declines in the estimated impact on idleness and having a learning disability, respectively, when we consider the impact on participants. The poor health estimates are relatively more stable; the reweighted impacts on participants are just $3 \%$ smaller than the FFE estimate.

In the final column of the table, we test whether the difference between the reweighted estimate for participants and the FFE estimate is statistically significant. We bootstrap the standard errors for this difference by taking draws with replacement from the sample and performing the FFE estimation and reweighting again. We do this 1,000 times and obtain the standard error of our difference as the standard deviation of the 1,000 estimated FFE-reweighted differences. We find that the reweighted estimates for some college (PSID) and high school graduation (CNLSY) are statistically different from the FFE estimate at the $5 \%$ and $10 \%$ levels, respectively. The remainder of the outcomes are more imprecisely estimated, and therefore we can not reject that the reweighted estimate is the same as the FFE estimate.

Returning to the PSID, Appendix Tables B.13 and B.14 show the PSID FFE estimates and reweighted results for high school and the good health index for whites, and the corresponding results for blacks. Overall, the results suggest little support for a positive long term effect of Head Start. This is true for the FFE estimates and the reweighted estimates. Nonetheless, the magnitude of the estimates can vary importantly with reweighting, particularly for whites. This makes sense since the identifying sample is a much smaller share of the overall sample for whites relative to blacks. For example, the FFE estimate for the good health index for whites is -0.27 $\mathrm{SD}$, but reweighting for the Head Start participant population changes this estimate to -0.34 . In contrast, the coefficients are relatively stable for blacks. ${ }^{61}$

We explore other reweighting strategies in Appendix Tables B.15 and B.16. Reweighting using linear extrapolation of treatment effects to singletons in Table B.15 produces qualitatively similar results to the baseline reweighting. ${ }^{62}$ Table B.16 presents the results when we reweight the FFE estimates using sample shares instead of propensity score weights. Across all outcomes, these estimates are quite similar to the FFE estimates, underscoring that the conditional-variance-weighting plays a minor role in this setting.

\footnotetext{
${ }^{61}$ For the black sample, most estimates are also statistically insignificant. However, for the age 30 Economic Sufficiency Index, the reweighted estimates indicate statistically significant negative impacts of Head Start. For example, for a target population of participants the reweighted coefficient on Head Start is -0.211 (s.e. $=0.073$ ).

${ }^{62}$ We have also explored excluding singletons altogether from the target. The estimates for non-singleton Head Start participants and non-singleton Head-Start-eligible children typically lie between the reweighted estimates for siblings and Head Start participants.
} 


\subsubsection{More Evidence on the Role of Family Size}

One key pattern in our findings is that larger families appear to have larger returns to Head Start than smaller families. We believe this to be a new finding in the Head Start literature. We note that this was not a pattern we initially set out to test in this study, so there is some chance of this finding being inadvertently driven by chance and our limited sample sizes. However we think that this may provide an interesting hypothesis for future studies. Also, we first observed this pattern in the PSID data, and so our CNLSY results are to some degree an out of sample confirmation of this pattern.

We have examined whether the larger coefficients for larger family sizes in Table 4 are driven by family size standing in for other covariates. In Appendix Table B.17 we perform a "horse race" analysis, comparing whether heterogeneous coefficients load on to family size, or other covariates. This table shows that the heterogeneity with family size is robust to also allowing for heterogeity along other covariates. We have also experimented with specifications that test for whether larger family size is merely proxying for "longer sibling cohort span," and do not find evidence that this is the case.

\subsubsection{Additional FFE Estimates}

Continuing our analysis of the PSID, we also investigate effects of Head Start on a variety of additional short-term outcomes, outcomes at age 40, as well as heterogeneity by race, gender by cohort in Appendix C. We do not find any systematic evidence of effects on any of these outcomes, or important heterogeneity along these dimensions.

\section{Other Applications}

We have shown empirical evidence for selection into identification for three FFE applications relating to the returns to human capital investment and returns to domestic migration. In each of these contexts, there appears to be a mechanical relationship between $\operatorname{Pr}\left(S_{g}=1\right)$ and group size. In the Head Start setting, heterogeneity along these lines creates an upward-bias in the FE estimate. Since returns to migration may also be heterogeneous by family characteristics, it may be useful to also reweight the estimates from Collins and Wanamaker (2014) to obtain the ATE for a representative set of migrants.

We now discuss three additional FE designs present in the education, labor, and environmental literatures that illustrate settings where the tools that we have developed may apply. First, a number of studies examine the effect of peers in the classroom within a school-grade (or school) using school-grade FE (or school FE). For example, Carrell and Hoekstra (2010) and Carrell, Hoekstra and Kuka (2018) examine the effect of having a peer exposed to domestic violence (DV) using this strategy, finding large negative impacts on contemporaneous achievement that persist to 
reduce long-term earnings. While the DV measure in these studies is continuous, it is reasonable to think that this may be a "lumpy" variable in the sense that some schools (or school-grades), which have a low probability of DV, will never have a student exposed to DV during the 8 year window of observation, and some school-grades, which have a high probability of DV, may always have a student exposed to DV. Given the likely correlation between $\operatorname{Pr}\left(D V_{g}=1\right)$ and $\operatorname{Pr}\left(S_{g}=1\right)$, non-switcher schools probably also have a different set of school resources (e.g. share of highly experienced teachers) and student composition (e.g. mean family income) than switchers, which could either exacerbate or mitigate the effects of DV. As a result, the effects estimated from switching schools may not generalize to low-probability-DV non-switchers or high-probability-DV non-switchers.

Second, a set of influential papers by Dube, Lester and Reich $(2010,2015)$ identify the impact of minimum wage laws within border-county pairs (using border-pair-by-year FE). This strategy produces bounds on minimum wage elasticities which are less negative than those estimated with other strategies. The authors report that $91 \%$ of the county pairs in the data have variation in the minimum wage at some point during the analysis, however states with more border counties and who have more frequent changes to the minimum wage relative to neighboring states will contribute more variation to the design. Hence, in practice, identification may be concentrated among a subset of the $91 \%$. At the same time, the characteristics of switching border counties are likely to be different from interior counties, in terms of the education distribution, population density, or industry composition, which could influence the response to minimum wage increases (Cengiz et al., 2019). Thus, reweighting the estimates of switching border pairs to account for these characteristics could yield a different estimate for the impact of the minimum wage.

Third, it has become common to estimate the effect of environmental shocks on health and human capital using variation in temperature or rainfall within a local area (e.g. district FE). For example, Shah and Steinberg (2017) employ this strategy and find that a positive rain shock (top $20 \%$ rainfall) reduces the likelihood that students attend school, and vice versa for droughts. Since shocks are by definition infrequent events, it is likely that some districts that have more moderate climates will have no shocks over the 4 years of analysis. These non-switching districts may be located in a different geography, have distinct industrial composition, or population characteristics, which could in turn affect the elasticity of school attendance. Hence, extrapolating from switcher to non-switcher districts may require reweighting strategies such as those we propose.

Some recent work notes empirical issues related to SI. In the context of estimating returns to experience for teachers, Wiswall (2013) observes that teacher-FE models, paired with modest panel lengths and experience categories such as " $4+$ years," imply that the coefficients on these categories will be solely identified from relatively new teachers, and lead to misestimation of the returns to experience. Spears, Coffey and Behrman (2019) raise SI concerns for FFE models used by Jayachandran and Pande (2017) to examine the relationship between birth order and child height in the Demographic and Health Surveys. Since these outcomes are only observed for those under 5 , 
Spears, Coffey and Behrman (2019) argue that a FE design selects for families with shorter birth spacing, which in turn may have more negative impacts of birth order.

As researchers using FE consider the relevance of SI for a particular setting, we also note that greater degrees of SI may, on the margin, favor a design that uses a more coarse level of fixed effects in order to increase the number of switchers. For example, Geruso and Spears (2018) examine the impacts of heat on infant mortality. They use district-by-calendar-month FEs in one specification, and village-by-calendar-month FEs as an alternative. The latter specification, with smaller groups, may have greater SI concerns. It also has larger standard errors, and the authors suggest that the coarser aggregation is their preferred model. The reweighting methods we propose could be employed to mitigate the SI concerns in applications that use more narrow FEs.

Overall, these applications highlight the fact that selection into identification is likely to be relevant across the numerous domains where FE are applied. We leave it to future researchers to quantify the role of this selection and apply reweighting techniques to test the sensitivity of the conclusions.

\section{Conclusion}

Fixed effects can provide a useful approach for treatment effect estimation. The internal validity of this strategy, which has been the subject of much debate, relies on the assumption that treatment is randomly assigned to units in a group. In this article, we show that an additional assumption is needed for the external validity of results: that groups with variation (switchers) have comparable treatment effects to groups without variation (non-switchers). In other words, fixed effects estimates are generalizable only if there is no selection into identification.

We show that this assumption is not trivial in the context of family fixed effects. We document across multiple settings that switching families are systematically larger and show that this can induce bias in estimation. We develop a novel approach to recover ATE's for representative populations, which upweights observations that are under-represented in the identifying sample relative to the population of interest. We demonstrate that this reweighting approach performs well using Monte Carlo simulations.

We apply these lessons to an analysis of the long term effects of Head Start in the PSID and CNLSY using family fixed effects. Relative to prior evaluations of Head Start using FFE in the PSID, we use a sample three times as large in size, include longer run (up to age 40) outcomes, and expand the set of outcomes under consideration. Echoing prior findings, we find using FFE that Head Start significantly increases the likelihood of completing some college and graduating from high school, and decreases the likelihood of being idle, having a disability, or reporting poor health.

Using our reweighting methods, we estimate that Head Start leads to a 2.1 p.p. increase in the likelihood of attending some college for Head Start participants, and a 5.2 p.p. increase for Head Start eligible. The ATE estimate for participants is $83 \%$ smaller than the FFE estimate, a difference 
which is statistically significant at the $5 \%$ level. We examine several other outcomes and find few statistically significant results. In sum, the FFE results in the PSID indicate that Head Start has little effect on many long term outcomes on average, with the exception of completing some college, and perhaps even detrimental effects for men. In the CNLSY, for high school graduation we find that the reweighted estimate for participants (4.8 p.p.) is $44 \%$ smaller than the FFE estimate, a difference which is statistically different at the $10 \%$ level. We find relatively less change associated with reweighting for other outcomes.

Overall, we interpret our findings as pointing primarily toward "increased uncertainty" and to a limited degree toward "zero effects" of the Head Start program. This suggests that there is some discordance between the long-term results from the FFE design, and new estimates using other designs, which generally produce larger and more robust effects of this intervention. Reconciling these findings is beyond the scope of this paper, but would be a productive avenue for future work.

Based on our findings, we propose new standards for practice when using FE or similar research designs to diagnose, and potentially correct for, the role of changes in sample composition in explaining the gap between OLS and FE estimates.

1. First, analyses should report the switching sample size in addition to the total sample size, including for relevant subsamples of the data (e.g. whites and blacks). It may also be useful to calculate the effective number of observations and share of identifying variation from true switchers to increase transparency into the variation among switchers.

2. Second, we suggest that researchers show a balance of observables across switching status to complement evidence of within-sample balance across treatment status. These covariates should include the number of units in a group (if there is imbalance) and correlates of treatment. For example, in the case of movers, one might consider testing for balance of urbanicity, age, and occupations. If there are differences in these covariates, researchers should examine heterogeneity along these dimensions. These tests are likely to have limited power to detect issues if there are interactions between covariates, but are a useful bellweather for important external validity concerns.

3. As a subsequent step, we recommend using propensity-score reweighting of the FE estimates to obtain estimates for a representative population or a policy-relevant population, such as program participants. Since these methods can perform unevenly under some models of heterogeneity, we suggest testing for sensitivity of results and reporting a range of estimates where applicable. 


\section{References}

Abrevaya, Jason. 2006. "Estimating the effect of smoking on birth outcomes using a matched panel data approach." Journal of Applied Econometrics, 21(4): 489-519.

Aizer, Anna, and Flavio Cunha. 2012. "The Production of Human Capital: Endowments, Investments and Fertility." National Bureau of Economic Research Working Paper 18429. DOI: $10.3386 / \mathrm{w} 18429$.

Almond, Douglas, Kenneth Y. Chay, and David S. Lee. 2005. "The Costs of Low Birth Weight." The Quarterly Journal of Economics, 120(3): 1031-1083.

Anderson, Michael L. 2008. "Multiple Inference and Gender Differences in the Effects of Early Intervention: A Reevaluation of the Abecedarian, Perry Preschool, and Early Training Projects." Journal of the American Statistical Association, 103.

Andersson, Fredrik, John C. Haltiwanger, Mark J. Kutzbach, Giordano E. Palloni, Henry O. Pollakowski, and Daniel H. Weinberg. 2016. "Childhood Housing and Adult Earnings: A Between-Siblings Analysis of Housing Vouchers and Public Housing." National Bureau of Economic Research Working Paper 22721.

Andrews, Isaiah, and Emily Oster. 2019. "A Simple Approximation for Evaluating External Validity Bias." Economics Letters, 178: 58-62. Working Paper.

Angrist, Joshua, and Jorn-Steffen Pischke. 2009. Mostly Harmless Econometrics. Princeton University Press.

Angrist, Joshua D. 1998. "Estimating the Labor Market Impact of Voluntary Military Service Using Social Security Data on Military Applicants." Econometrica, 66(2): 249-288.

Angrist, Joshua D., and Ivan Fernandez-Val. 2013. "ExtrapoLATE-ing: External Validity and Overidentification in the LATE Framework." Advances in Economics and Econometrics: Tenth World Congress, , ed. Daron Acemoglu, Manuel Arellano and Eddie Dekel Vol. 3 of Econometric Society Monographs, 401 - 434. Cambridge University Press.

Aronow, Peter M., and Allison Carnegie. 2013. "Beyond LATE: Estimation of the Average Treatment Effect with an Instrumental Variable." Political Analysis, 21(4): 492-506.

Bailey, Martha J., Shuqiao Sun, and Brenden Timpe. 2018. "Prep School for Poor Kids: The Long-Run Impacts of Head Start on Human Capital and Economic Self-Sufficiency." Working Paper.

Barr, Andrew, and Chloe R. Gibbs. 2018. "Breaking the Cycle? Intergenerational Effects of an Anti-Poverty Program in Early Childhood." mimeo. 
Bates, Michael David, Katherine E. Castellano, Sophia Rabe-Hesketh, and Anders Skrondal. 2014. "Handling Correlations Between Covariates and Random Slopes in Multilevel Models." Journal of Educational and Behavioral Statistics, 39(6): 524-549.

Bauer, Lauren, and Diane Whitmore Schanzenbach. 2016. "The Long-Term Impact of the Head Start Program." The Hamilton Project.

Bayer, Patrick, Randi Hjalmarsson, and David Pozen. 2009. "Building Criminal Capital behind Bars: Peer Effects in Juvenile Corrections*." The Quarterly Journal of Economics, 124(1): 105-147.

Beck, Nathaniel. 2015. "Estimating Grouped Data Models with a Binary Dependent Variable and Fixed Effects: What are the Issues? Comments Prepared for Delivery at the Annual Meeting of the Society for Political Methodology." mimeo.

Black, Sandra E., Paul J. Devereux, and Kjell G. Salvanes. 2007. "From the Cradle to the Labor Market? The Effect of Birth Weight on Adult Outcomes*." The Quarterly Journal of Economics, 122(1): 409-439.

Borusyak, Kirill, and Xavier Jaravel. 2017. "Revisiting Event Study Designs, with an Application to the Estimation of the Marginal Propensity to Consume." Working Paper.

Bound, John, and Gary Solon. 1999. "Double Trouble: On the Value of Twins-based Estimation of the Return to Schooling." Economics of Education Review, 18(2): 169-182.

Callaway, Brantly, and Pedro H. C. Sant'Anna. 2018. "Difference-in-Differences with Multiple Time Periods and an Application on the Minimum Wage and Employment." Working Paper.

Cameron, A. Colin, and Pravin K. Trivedi. 2005. Microeconometrics: Methods and Applications. Cambridge University Press.

Carneiro, Pedro, and Rita Ginja. 2014. "Long-Term Impacts of Compensatory Preschool on Health and Behavior: Evidence from Head Start." American Economic Journal: Economic Policy, 6(4): 135-173.

Carrell, Scott E., and Mark L. Hoekstra. 2010. "Externalities in the Classroom: How Children Exposed to Domestic Violence Affect Everyone's Kids." American Economic Journal: Applied Economics, 2(1): 211-228.

Carrell, Scott E., Mark Hoekstra, and Elira Kuka. 2018. "The Long-Run Effects of Disruptive Peers." American Economic Review, 108(11): 3377-3415.

Cengiz, Doruk, Arindrajit Dube, Attila Lindner, and Ben Zipperer. 2019. "The Effect of Minimum Wages on Low-Wage Jobs*." The Quarterly Journal of Economics. 
Chaisemartin, Clement, and Xavier D'Haultfoeille. 2019. "Two-way Fixed Effects Estimators with Heterogeneous Treatment Effects." mimeo.

Chamberlain, Gary. 1980. "Analysis of Covariance with Qualitative Data." The Review of Economic Studies, 47(1): 225-238.

Chernozhukov, Victor, IvÃ $i n$ FernÃ $i n d e z-V a l$, Jinyong Hahn, and Whitney Newey. 2013. "Average and Quantile Effects in Nonseparable Panel Models." Econometrica, 81(2): 535580.

Chetty, Raj, and Nathaniel Hendren. 2018a. "The Impacts of Neighborhoods on Intergenerational Mobility I: Childhood Exposure Effects*." The Quarterly Journal of Economics, 133(3): 1107-1162.

Chetty, Raj, and Nathaniel Hendren. 2018b. "The Impacts of Neighborhoods on Intergenerational Mobility II: County-Level Estimates." The Quarterly Journal of Economics, 133(3): 11631228.

Chorniy, Anna V, Janet Currie, and Lyudmyla Sonchak. 2018. "Does Prenatal WIC Participation Improve Child Outcomes?" National Bureau of Economic Research Working Paper 24691.

Collins, William J., and Marianne H. Wanamaker. 2014. "Selection and Economic Gains in the Great Migration of African Americans: New Evidence from Linked Census Data." American Economic Journal: Applied Economics, 6(1): 220-252.

Currie, Janet, and Duncan Thomas. 1995. "Does Head Start Make a Difference?" American Economic Review, 85(3): 341-364.

Currie, Janet, and Ishita Rajani. 2015. "Within-Mother Estimates of the Effects of WIC on Birth Outcomes in New York City." Economic Inquiry, 53(4): 1691-1701.

Currie, Janet, and Maya Rossin-Slater. 2013. "Weathering the storm: Hurricanes and birth outcomes." Journal of Health Economics, 32(3): 487 - 503.

Deming, David. 2009. "Early Childhood Intervention and Life-Cycle Skill Development: Evidence from Head Start." American Economic Journal: Applied Economics, 1(3): 111-134.

Dube, Arindrajit, T. William Lester, and Michael Reich. 2010. "Minimum Wage Effects Across State Borders: Estimates Using Contiguous Counties." The Review of Economics and Statistics, 92(4): 945-964.

Dube, Arindrajit, T. William Lester, and Michael Reich. 2015. "Minimum Wage Shocks, Employment Flows, and Labor Market Frictions." Journal of Labor Economics, 34(3): 663-704. 
Fernandez-Val, Ivan. 2009. "Fixed Effects Estimation of Structural Parameters and Marginal Effects in Panel Probit Models." Journal of Econometrics, 150(1): 71 - 85.

Figlio, David, Jonathan Guryan, Krzysztof Karbownik, and Jeffrey Roth. 2014. "The Effects of Poor Neonatal Health on Children's Cognitive Development." American Economic Review, 104(12): 3921-3955.

Finkelstein, Amy, Matthew Gentzkow, and Heidi Williams. 2016. "Sources of Geographic Variation in Health Care: Evidence From Patient Migration*." The Quarterly Journal of Economics, 131(4): 1681-1726.

Garces, Eliana, Duncan Thomas, and Janet Currie. 2002. "Longer-Term Effects of Head Start." American Economic Review, 92(4): 999-1012.

Geruso, Michael, and Dean Spears. 2018. "Heat, Humidity, and Infant Mortality in the Developing World.” National Bureau of Economic Research Working Paper 24870.

Gibbons, Charles E., Serrato Juan Carlos Suarez, and Michael B. Urbancic. 2018. "Broken or Fixed Effects?" Journal of Econometric Methods, 0(0).

Gibbs, Chloe, Jens Ludwig, and Douglas L Miller. 2013. "Does Head Start Do Any Lasting Good?" Legacies of the War on Poverty, , ed. Martha J. Bailey and Sheldon Danziger. Russell Sage Foundation.

Goodman-Bacon, Andrew. 2018. "Difference-in-Differences with Variation in Treatment Timing." National Bureau of Economic Research Working Paper 25018.

Hoxby, Caroline M. 2000. "The Effects of Class Size on Student Achievement: New Evidence from Population Variation." Quarterly Journal of Economics, 115(4): 1239-1285.

Hoynes, Hilary, Diane Whitmore Schanzenbach, and Douglas Almond. 2016. "Long-Run Impacts of Childhood Access to the Safety Net." American Economic Review, 106(4): 903-934.

Imai, Kosuke, and In Song Kim. 2019. "When Should We Use Unit Fixed Effects Regression Models for Causal Inference with Longitudinal Data?" American Journal of Political Science, 63(2): 467-490.

Imbens, Guido W., and Donald B. Rubin. 2015. Causal Inference for Statistics, Social, and Biomedical Sciences: An Introduction. Cambridge University Press.

Jayachandran, Seema, and Rohini Pande. 2017. "Why Are Indian Children So Short? The Role of Birth Order and Son Preference." American Economic Review, 107(9): 2600-2629. 
Johnson, Rucker C., and C. Kirabo Jackson. 2017. "Reducing Inequality Through Dynamic Complementarity: Evidence from Head Start and Public School Spending." National Bureau of Economic Research Working Paper 23489. DOI: 10.3386/w23489.

Kling, Jeffrey R, Jeffrey B Liebman, and Lawrence F Katz. 2007. "Experimental Analysis of Neighborhood Effects." Econometrica, 75(1): 83-119.

Lemieux, Thomas. 1998. "Estimating the Effects of Unions on Wage Inequality in a Panel Data Model with Comparative Advantage and Nonrandom Selection." Journal of Labor Economics, 16(2): 261-291.

Leuven, Edwin, and Monique de Haan. 2016. "Head Start and the Distribution of Long Term Education and Labor Market Outcomes." IZA Institute of Labor Economics Working Paper.

Lochner, Lance, and Enrico Moretti. 2015. "Estimating and Testing Models with Many Treatment Levels and Limited Instruments." The Review of Economics and Statistics, 97(2): 387-397.

Loken, Katrine V., Magne Mogstad, and Matthew Wiswall. 2012. "What Linear Estimators Miss: The Effects of Family Income on Child Outcomes." American Economic Journal: Applied Economics, 4(2): 1-35.

Ludwig, Jens, and Douglas L. Miller. 2007. "Does Head Start Improve Children's Life Chances? Evidence from a Regression Discontinuity Design." The Quarterly Journal of Economics, 122(1): 159-208.

Mundlak, Yair. 1978. "On the Pooling of Time Series and Cross Section Data." Econometrica, 46(1): 69-85.

Pages, Remy J.-C., Dylan J. Lukes, Drew H. Bailey, and Greg J. Duncan. 2019. "Elusive Longer-Run Impacts of Head Start: Replications Within and Across Cohorts." arXiv:1903.01954 [econ, q-fin]. arXiv: 1903.01954.

Robins, James M., Andrea Rotnitzky, and Lue Ping Zhao. 1995. "Analysis of Semiparametric Regression Models for Repeated Outcomes in the Presence of Missing Data." Journal of the American Statistical Association, 90(429): 106-121.

Rossin-Slater, Maya. 2013. "WIC in your neighborhood: New evidence on the impacts of geographic access to clinics." Journal of Public Economics, 102: 51-69.

Roy, A. D. 1951. "Some Thoughts on the Distribution of Earnings." Oxford Economic Papers, $3(2): 135-146$.

Shah, Manisha, and Bryce Millett Steinberg. 2017. "Drought of Opportunities: Contemporaneous and Long-Term Impacts of Rainfall Shocks on Human Capital." Journal of Political Economy, 125(2): 527-561. 
Sloczynski, Tymon. 2018. "A General Weighted Average Representation of the Ordinary and Two-Stage Least Squares Estimands." Working Paper.

Spears, Dean, Diane Coffey, and Jere Behrman. 2019. "Birth Order, Fertility, and Child Height in India and Africa." National Bureau of Economic Research Working Paper 12289.

Stuart, Elizabeth A., Stephen R. Cole, Catherine P. Bradshaw, and Philip J. Leaf. 2011. "The Use of Propensity Scores to Assess the Generalizability of Results from Randomized Trials." Journal of the Royal Statistical Society: Series A (Statistics in Society), 174(2): 369-386.

Suri, Tavneet. 2011. "Selection and Comparative Advantage in Technology Adoption." Econometrica, 79(1): 159-209.

Thompson, Owen. 2017. "Head Start's Long-Run Impact: Evidence from the Program's Introduction." Journal of Human Resources.

Verdier, Valentin, and Andrew Castro. 2019. "Average Treatment Effects for Stayers with Correlated Random Coefficient Models of Panel Data." mimeo.

Wiswall, Matthew. 2013. "The dynamics of teacher quality." Journal of Public Economics, 100: $61-78$.

Wooldridge, Jeffrey M. 2005. "Fixed-Effects and Related Estimators for Correlated RandomCoefficient and Treatment-Effect Panel Data Models." The Review of Economics and Statistics, 87(2): 385-390.

Wooldridge, Jeffrey M. 2010. Econometric Analysis of Cross Section and Panel Data. MIT Press.

Wooldridge, Jeffrey M. 2019. "Correlated random effects models with unbalanced panels." Journal of Econometrics, 211(1): 137 - 150. Annals Issue in Honor of Jerry A. Hausman.

Xie, Zong-Xian, Shin-Yi Chou, and Jin-Tan Liu. 2016. "The Short-Run and Long-Run Effects of Birth Weight: Evidence from Large Samples of Siblings and Twins in Taiwan." Health Economics, 26(7): 910-921. 


\section{$9 \quad$ Figures}

Figure 1: Within-Family Variation in Head Start and Attendance of Some College (PSID)

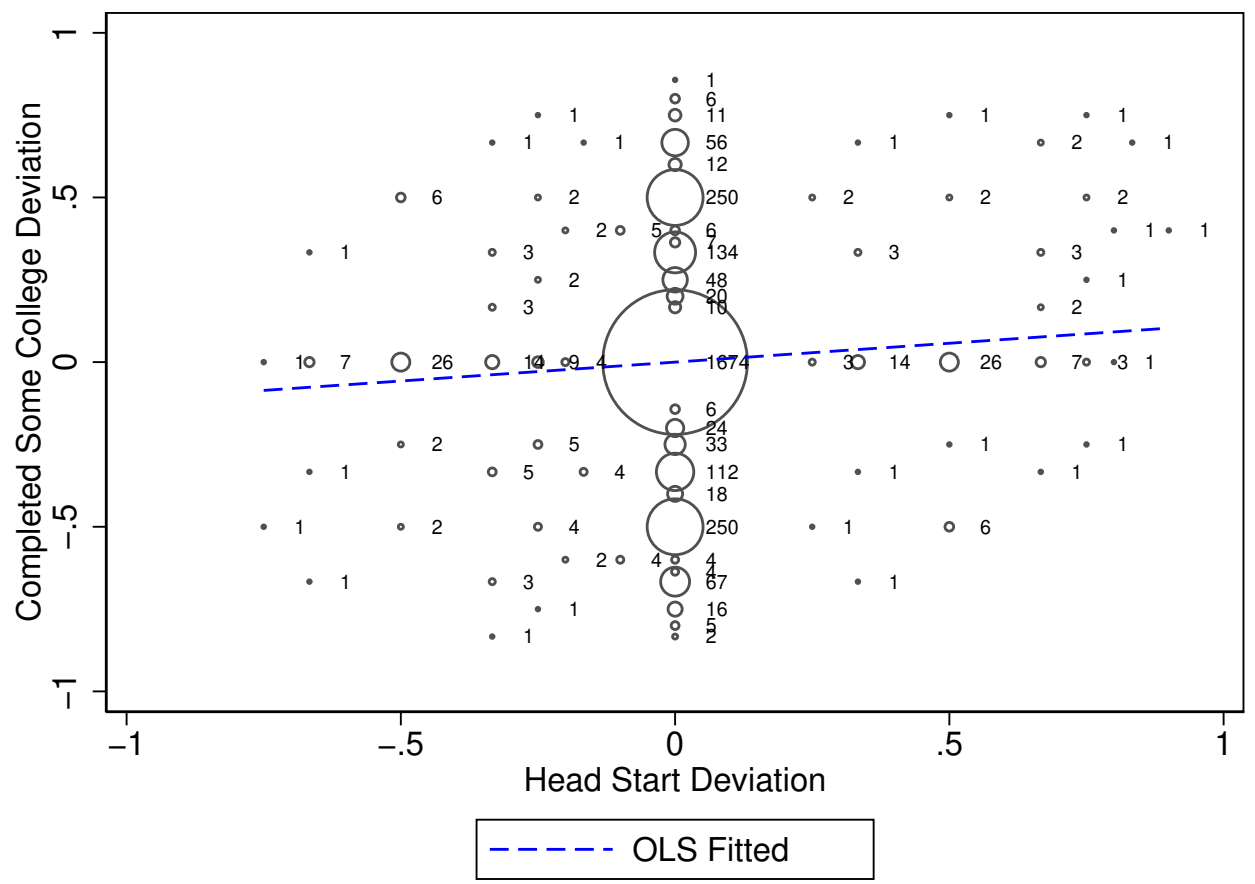

Notes: This figure depicts the identifying variation used in a FFE regression of some college on an indicator for participation in Head Start. Each marker represents the number of individuals that exhibit a particular deviation from the mean Head Start attendance of their family and from the mean attendance of some college of their family. Deviations are defined as the difference between individual attendance of Head Start/some college (1 or 0$)$ and mean of Head Start/some college of one's family. The marker size represents the unweighted number of individuals. We also include a best-fit line, weighted by the number of individuals in each marker. Source: Panel Study of Income Dynamics, 1968-2011 waves. 
Figure 2: Likelihood of Being a Switcher Family Increases with Family Size and P(treatment)

(a) Head Start in PSID

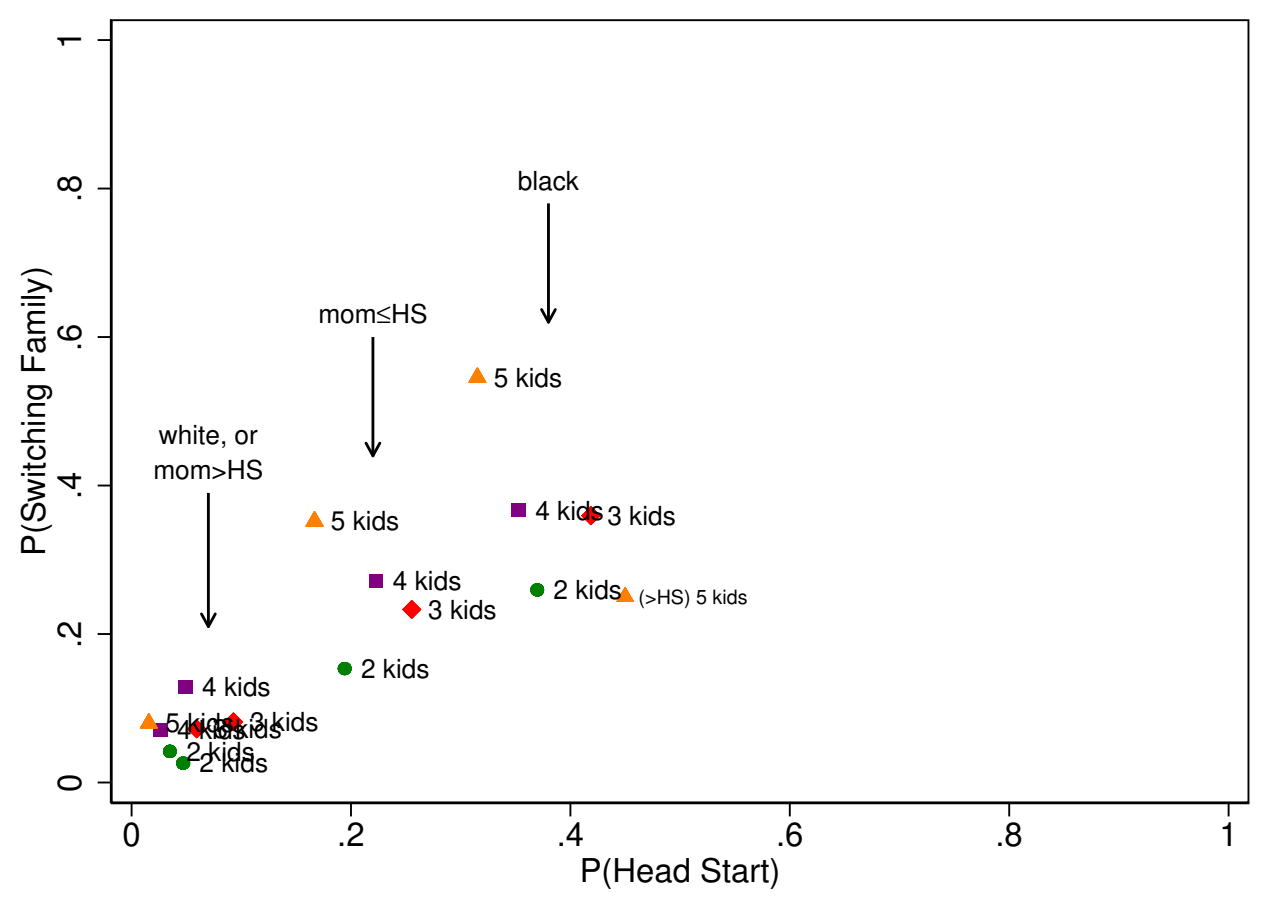

(b) Head Start in CNLSY

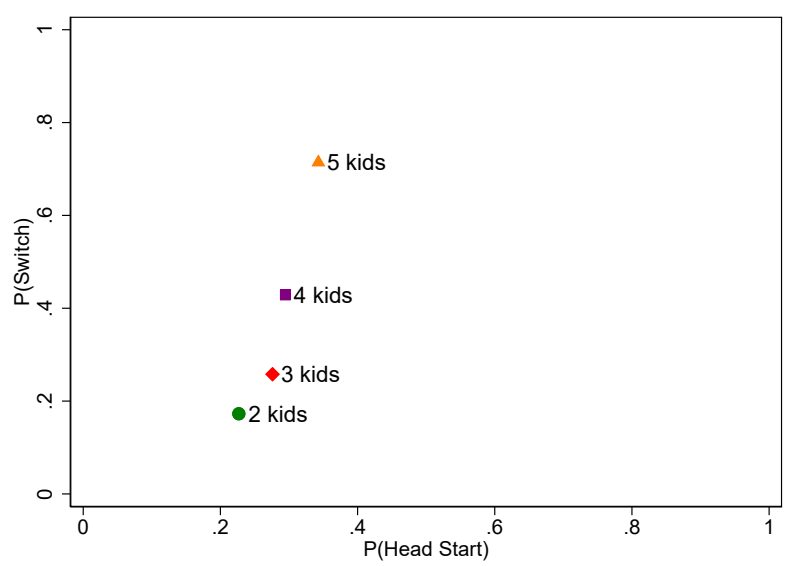

(c) Migration in Census

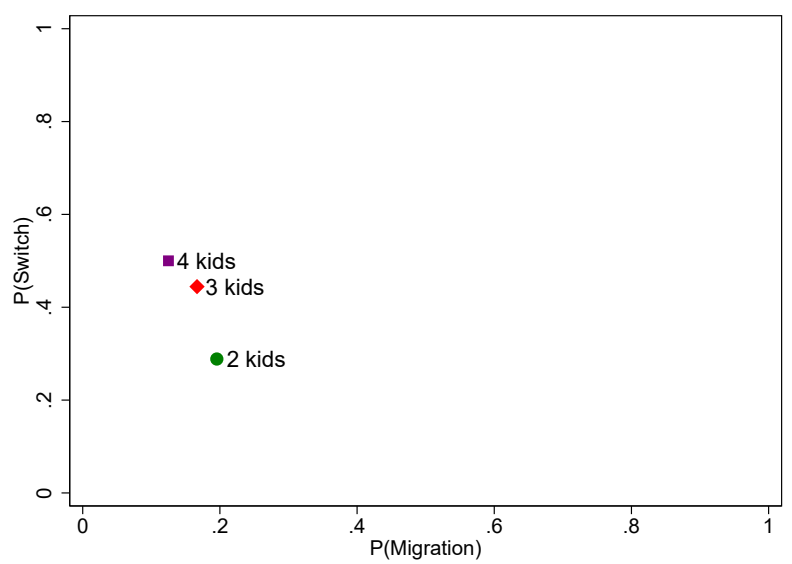

Notes: This figure shows the probability of being in a switching family and the probability of "treatment" by family size using three datasets and varying treatments. Panel (a) plots the probability of being in a switching family and of attending Head Start by family size for the following groups in the PSID: Whites, Blacks, children of mothers with at most a high school degree, and children of mothers with at least some college. Figure (b) is a simplified version of (a) using data on Head Start participation and family size from the CNLSY. Figure (c) shows the probability of being in a switching family and the probability of migrating to the northern US, using a linking of the 1910 to 1930 censuses used in Collins and Wanamaker (2014). 
Figure 3: FFE Weights and Head-Start-Participant-Representative Weights by Family Size and Some College $\beta$ (PSID White Sample)

(a) Families with 2-3 Children

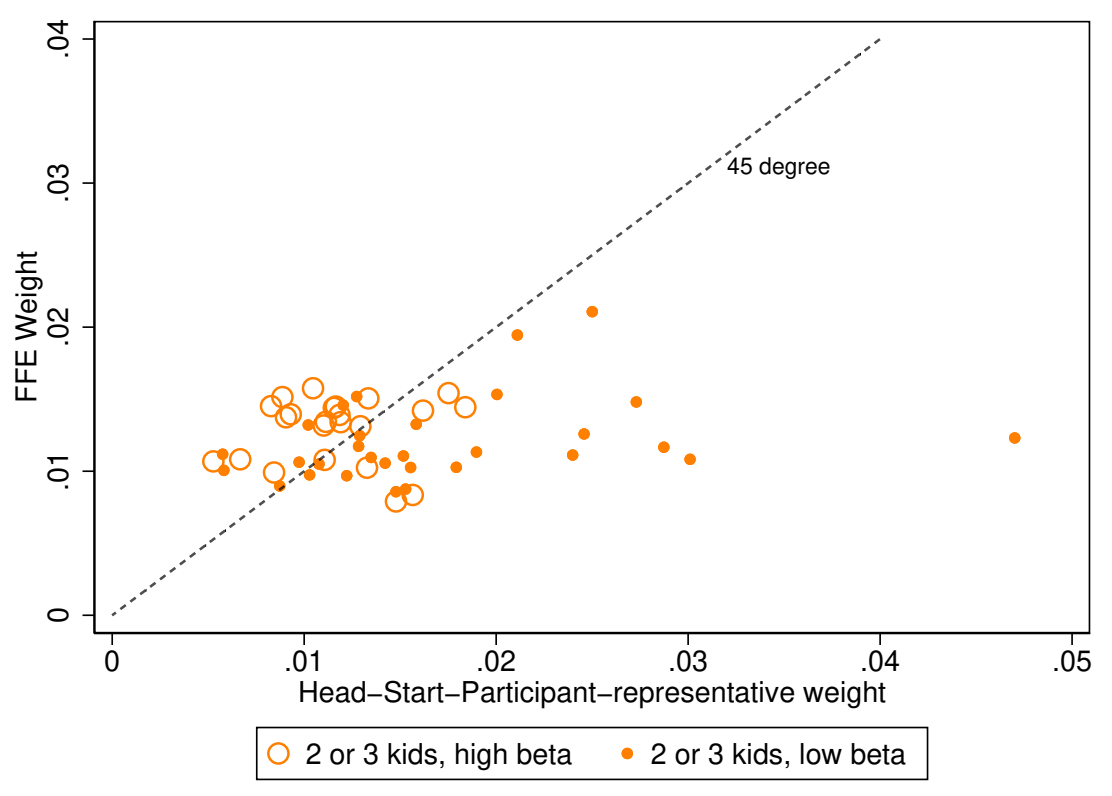

(b) Families with 4+ Children

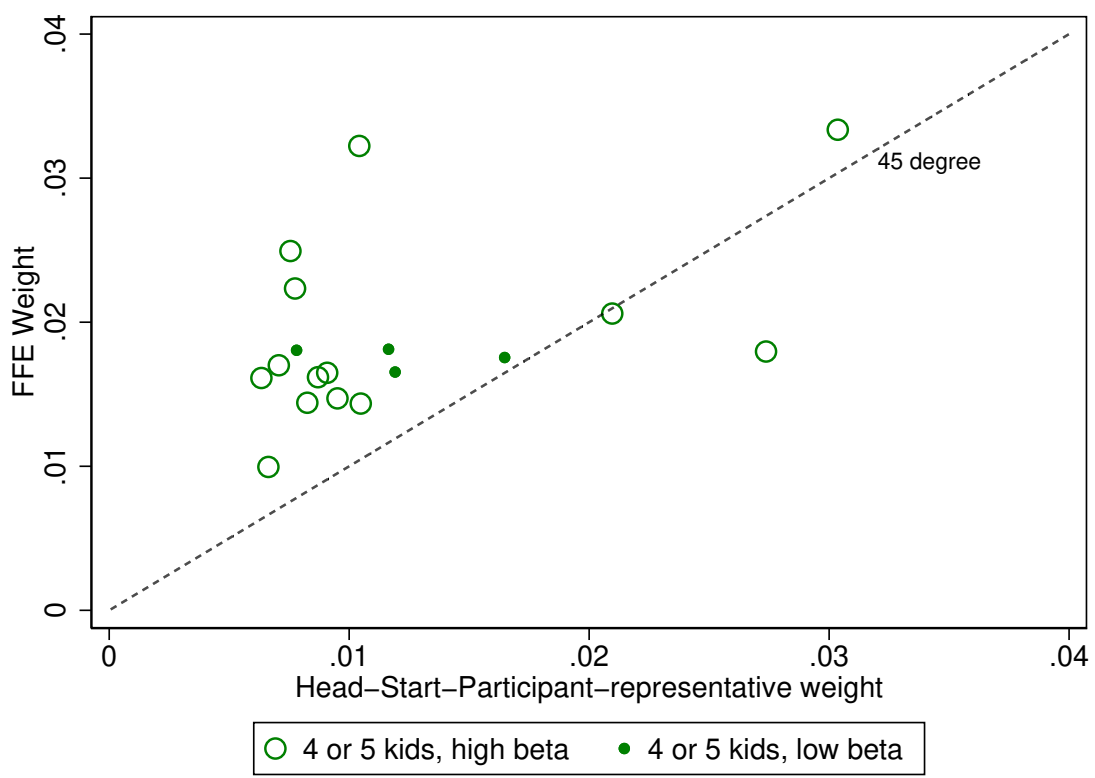

Notes: Each marker in this figure indicates the FFE weights and Head-Start-participantrepresentative (post-regression) weight for one white switching family. The color of the marker indicates whether the family has 2-3 children or 4 or more children. The size of the marker indicates the estimated family-specific beta from a regression of attainment of some college on interactions between Head Start and family id fixed effects. A larger marker indicates an above median beta, while a smaller marker indicates a below-median beta. The 45 degree line is included for reference. Observations above (below) the line are overweighted (underweighted) in the FFE sample relative to a representative Head Start sample. Source: Parnel Study of Income Dynamics, 1968-2011 waves. 


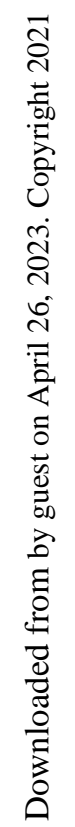




\section{Tables}

Table 1: Family FE Articles in Top Applied Journals, 2002 to 2017

\begin{tabular}{|c|c|c|c|c|}
\hline & Binary Indep. & Binary Dep. & Both Binary & Total \\
\hline AEJ: Applied & 6 & 4 & 3 & 8 \\
\hline AEJ: Economic Policy & 1 & 1 & 1 & 1 \\
\hline AER & 3 & 1 & 1 & 5 \\
\hline AER Papers and Proceedings & 2 & 2 & 1 & 3 \\
\hline Journal of Health Economics & 5 & 3 & 2 & 7 \\
\hline Journal of Human Resources & 7 & 2 & 2 & 12 \\
\hline Journal of Labor Economics & 2 & 1 & 1 & 5 \\
\hline Journal of Political Economy & 2 & 1 & 1 & 2 \\
\hline Journal of Public Economics & 4 & 4 & 4 & 5 \\
\hline QJE & 1 & 4 & 1 & 4 \\
\hline Review of Economics and Statistics & 2 & 0 & 0 & 3 \\
\hline Total & 35 & 23 & 17 & 55 \\
\hline \multicolumn{5}{|l|}{ Common Dependent Variables } \\
\hline Schooling/Attainment & 23 & & & \\
\hline Test Score & 17 & & & \\
\hline Employment/Earnings & 15 & & & \\
\hline Birth Weight & 6 & & & \\
\hline Health & 6 & & & \\
\hline Behavioral Issues/Crime & 5 & & & \\
\hline \multicolumn{5}{|l|}{ Common Independent Variables } \\
\hline$\overline{\text { Schooling }}$ & 8 & & & \\
\hline Birth Weight & 5 & & & \\
\hline Health & 5 & & & \\
\hline Parental Traits & 4 & & & \\
\hline Employment & 3 & & & \\
\hline Birth order & 3 & & & \\
\hline Means-Tested Public Program & 2 & & & \\
\hline Death of Family Member & 2 & & & \\
\hline Bombing/Radiation & 2 & & & \\
\hline \multicolumn{5}{|l|}{ Observations by Sample } \\
\hline & Siblings N & Total N & & \\
\hline $\mathrm{p} 10$ & 469 & 1,212 & & \\
\hline $\mathrm{p} 25$ & 1,167 & 2,142 & & \\
\hline $\mathrm{p} 50$ & 6,315 & 17,501 & & \\
\hline p75 & 160,122 & 551,630 & & \\
\hline p90 & 750,697 & $1,582,142$ & & \\
\hline Year Publication Min/Max & 2002 & 2017 & & \\
\hline Articles with Balance Table if Binary Indep. & 1 & & & \\
\hline
\end{tabular}

Notes: This table presents a summary of FFE articles published between January 2000 and May 2017 in 11 top applied journals, which are listed in the first panel of the table. For reference, between 2002 and 2017 the number of articles published in AEJ: Applied was 310; AEJ: Policy was 313; AER was 1722; AER P\&P was 1676; JoLE was 434; Journal of Political Economy was 548; QJE was 639; JHR was 543; JPubE was 1688; REStat was 1033; JHE was 1017. Articles were initially idenitified using the search terms "family," "within family," "sibling," "twin," "mother," "father," "brother," "sister," fixed effect," "fixed-effect," and "birthweight" using queries on journal websites. Siblings N is the number of obsertations reported for the sample of siblings, while Total $\mathrm{N}$ represents the number of total observations reported. See text for details. 
Table 2: Switchers and Non-Switchers Vary Along Dimensions Other Than Family Size

\begin{tabular}{|c|c|c|c|c|c|}
\hline & $\begin{array}{c}(1) \\
\text { Switch } \\
\end{array}$ & $\begin{array}{c}(2) \\
\text { Non-Switch } \\
\end{array}$ & $\begin{array}{c}(3) \\
\text { P-Val. }(1)=(2)\end{array}$ & $\begin{array}{c}(4) \\
\text { Beta Switch }\end{array}$ & $\begin{array}{c}(5) \\
\text { P-Val (4) } \\
\end{array}$ \\
\hline \multicolumn{6}{|l|}{ A. Individual Covariates } \\
\hline$\overline{\text { Fraction female }}$ & 0.562 & 0.495 & 0.001 & 0.024 & 0.472 \\
\hline Fraction African-American & 0.516 & 0.111 & 0.000 & 0.249 & 0.000 \\
\hline Mother's yrs education & 9.283 & 11.230 & 0.000 & -0.140 & 0.453 \\
\hline Father's yrs education & 9.190 & 11.371 & 0.000 & -0.389 & 0.075 \\
\hline Had a single mother at age 4 & 0.252 & 0.099 & 0.000 & 0.055 & 0.011 \\
\hline Family income (age 3-6) (CPI adjusted) & 31809 & 52574 & 0.000 & -4759 & 0.000 \\
\hline Mother employed, age 0 & 0.508 & 0.570 & 0.013 & 0.055 & 0.019 \\
\hline Mother employed, age 1 & 0.517 & 0.543 & 0.281 & 0.058 & 0.018 \\
\hline Mother employed, age 2 & 0.536 & 0.554 & 0.439 & 0.118 & 0.000 \\
\hline Household size at age 4 & 5.487 & 4.451 & 0.000 & 0.755 & 0.000 \\
\hline Fraction low birth weight & 0.077 & 0.058 & 0.075 & 0.010 & 0.483 \\
\hline Observations & 1103 & 5500 & 6603 & 7372 & 7372 \\
\hline \multicolumn{6}{|l|}{ B. Inverse Selection into Identification Wts. } \\
\hline $\operatorname{Pr}($ switch $) / \operatorname{Pr}($ Head Start $)$, Whites & $\begin{array}{l}2.993 \\
(2.17)\end{array}$ & $\begin{array}{l}2.347 \\
(1.95)\end{array}$ & & & \\
\hline $\operatorname{Pr}($ switch $) / \operatorname{Pr}($ Head Start $)$, Blacks & $\begin{array}{c}1.969 \\
(1.29)\end{array}$ & $\begin{array}{l}1.137 \\
(1.03)\end{array}$ & & & \\
\hline
\end{tabular}

Notes: Panel A of this table presents comparisons of the characteristics of individuals in switching families and non-switching families. Columns 1, 2, and 3, respectively, show the mean characteristics of individuals in families that are switchers; individuals in families that are not switchers; and individuals that attended Head Start (HS) in non-switcher families. Column 3 presents the p-value for the test that columns 1 and 2 are equal. Column 4 shows the estimates from a regression of each row heading on an indicator for being in a switcher family, with the corresponding p-value shown in Column 5, with standard errors clustered on id1968. All controls from the main specification are included, except the variable shown in the row heading. All estimates are weighted to be representative of 1995 population; see text for details. Panel B shows the mean and standard deviation (in parenthesis) of the inverse of the post-regression propensity score weights when the target is Head Start participants. This gives a measure of how aligned the characteristics of switchers are with the characteristics of Head Start participants, the population of interest. An average value of 1 implies perfect alignment, while a higher value implies that the characteristics of switchers are over-represented relative to the characteristics of Head Start participants. $\operatorname{Pr}(\mathrm{switch})$ and $\operatorname{Pr}(\mathrm{Head}$ Start) are estimated from a multinomial logit model of these outcomes on family size and other covariates described in the text. Source: Panel Study of Income Dynamics, 1968-2011 waves. 
Table 3: Change in Weighting of Regression Estimates Across Sibling and Switcher Samples (PSID)

\begin{tabular}{lccccc}
\hline & \multicolumn{5}{c}{ Number of Children in Family: } \\
\cline { 2 - 6 } & 1 & 2 & 3 & 4 & $5+$ \\
\hline \hline A. Share of Sample & & & & & \\
\hline All Sample (No FFE) & 0.123 & 0.273 & 0.238 & 0.147 & 0.134 \\
Siblings Sample (No FFE) & 0.000 & 0.345 & 0.300 & 0.186 & 0.169 \\
Switchers Sample (FFE) & 0.000 & 0.210 & 0.271 & 0.197 & 0.322 \\
\hline B. Variance in Head Start & & & & & \\
All Sample (No FFE) & 0.089 & 0.104 & 0.121 & 0.127 & 0.132 \\
Siblings Sample (No FFE) & 0.000 & 0.024 & 0.050 & 0.059 & 0.068 \\
Switchers Sample (FFE) & 0.000 & 0.045 & 0.098 & 0.131 & 0.174 \\
\hline C. Regression weights & & & & & \\
\hline All Sample (No FFE) & 0.171 & 0.257 & 0.284 & 0.117 & 0.101 \\
Siblings Sample (No FFE) & 0.000 & 0.338 & 0.374 & 0.154 & 0.134 \\
Switchers Sample (FFE) & 0.000 & 0.256 & 0.307 & 0.190 & 0.248 \\
\hline \hline
\end{tabular}

Notes: This table shows the change in the composition of the PSID sample moving from all individuals and estimating a model without controls ("All Sample (no FFE)"); to individuals that have at least one other sibling in the sample and estimating a model without controls ("Siblings Sample (no FFE)"); to individuals in families that have variation in Head Start attendance and estimating a model with family fixed effects ("Switchers sample (FFE)" ) Panel A shows the share of individuals in each sample that come from a family with 1 child (zero siblings), 2 children, etc. Panel B shows the variance in Head Start for each family size and sample. For the switcher sample, this is calculated net of family fixed effects. Panel C shows the "regression weight" given to each family size in a given sample, denoted as $\omega_{z}$ and defined formally in Section 3. The shares and regression weights do not sum to one for the "all sample" because this sample also includes an additional category of individuals who have an unknown number of siblings (due to a missing mother id). These individuals account for 8.5 percent of the "all sample." Source: Panel Study of Income Dynamics, 1968-2011 waves. 
Table 4: Returns to Head Start by Family Size, and Implications for Regression Estimates

\begin{tabular}{|c|c|c|c|c|c|}
\hline & \multirow{2}{*}{\multicolumn{2}{|c|}{$\begin{array}{c}\text { PSID } \\
\text { Some College }\end{array}$}} & \multicolumn{3}{|c|}{ CNLSY } \\
\hline & & & \multirow{2}{*}{$\frac{\text { HS Grad }}{\text { FE }}\left(\begin{array}{c}(3)\end{array}\right.$} & \multirow{2}{*}{$\begin{array}{c}\text { Idle } \\
\text { FE } \\
(4)\end{array}$} & \multirow{2}{*}{$\begin{array}{c}\text { Lrn. Disab. } \\
\text { FE } \\
(5)\end{array}$} \\
\hline & $\begin{array}{l}\text { CX } \\
(1)\end{array}$ & $\begin{array}{l}\mathrm{FE} \\
(2)\end{array}$ & & & \\
\hline \multicolumn{6}{|l|}{ A. Effects by Family Size } \\
\hline 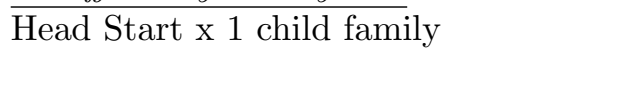 & $\begin{array}{l}0.169^{*} \\
(0.091)\end{array}$ & & & & \\
\hline Head Start x 2 child family & $\begin{array}{c}0.038 \\
(0.079)\end{array}$ & $\begin{array}{l}-0.126 \\
(0.099)\end{array}$ & $\begin{array}{c}0.058 \\
(0.050)\end{array}$ & $\begin{array}{l}-0.075 \\
(0.060)\end{array}$ & $\begin{array}{l}-0.018 \\
(0.025)\end{array}$ \\
\hline Head Start x 3 child family & $\begin{array}{l}-0.030 \\
(0.087)\end{array}$ & $\begin{array}{l}0.152^{* *} \\
(0.075)\end{array}$ & $\begin{array}{c}0.042 \\
(0.063)\end{array}$ & $\begin{array}{l}-0.001 \\
(0.071)\end{array}$ & $\begin{array}{l}-0.073 \\
(0.046)\end{array}$ \\
\hline Head Start x 4 child family & $\begin{array}{l}-0.053 \\
(0.100)\end{array}$ & $\begin{array}{c}0.251^{* * *} \\
(0.091)\end{array}$ & $\begin{array}{c}0.135 \\
(0.087)\end{array}$ & $\begin{array}{l}-0.063 \\
(0.118)\end{array}$ & $\begin{array}{l}-0.042 \\
(0.052)\end{array}$ \\
\hline Head Start x $5+$ child family & $\begin{array}{c}0.572^{* * *} \\
(0.119)\end{array}$ & $\begin{array}{c}0.348^{* * *} \\
(0.126)\end{array}$ & $\begin{array}{c}0.305^{* * *} \\
(0.095)\end{array}$ & $\begin{array}{c}-0.317^{* *} \\
(0.132)\end{array}$ & $\begin{array}{c}-0.161^{*} \\
(0.091)\end{array}$ \\
\hline Head Start x Unknown child family & $\begin{array}{l}-0.099 \\
(0.108)\end{array}$ & & & & \\
\hline Observations & 4258 & 2986 & 1251 & 1251 & 1247 \\
\hline Head Start Switchers & & 213 & 668 & 668 & 668 \\
\hline Effective Obs. (Indivs. 2-Person Fams) & & 235.9 & 644.3 & 644.3 & 644.3 \\
\hline Effective Obs. (CX Indivs.) & & 731.8 & 558.9 & 558.9 & 558.9 \\
\hline \multicolumn{6}{|l|}{$\frac{\text { B. Simulated Estimates across Samples }}{\text { using Family-Size Regression Weights }}$} \\
\hline$\overline{\text { All }}$ & 0.046 & & & & \\
\hline Siblings & 0.037 & 0.083 & 0.081 & -0.071 & -0.047 \\
\hline Switchers & 0.069 & 0.123 & 0.093 & -0.077 & -0.054 \\
\hline
\end{tabular}

Notes: Panel A of this table shows the coefficients from regressions of outcomes on a series of indicators for whether an individual attended Head Start interacted with an indicator for the number of children in one's family. The data source and specification varies across columns. Columns 1 and 2 use our main PSID sample and the outcome is attainment of some college. Columns 3-5 use the CNLSY79 sample and the outcomes are indicators for graduating from high school, being idle, and having a learning disability, respectively. Columns 1 include controls, but not mother f.e., and standard errors are clustered at the family id level. Columns 2-5 include mother fixed effects, and standard errors clustered by mother id. The number of Head Start switchers is equal to the number of individuals in families that have variation in Head Start. "Effective Obs. (CX Indivs.)" is the equivalent number of cross-sectional units that provide the same amount of variation as switchers. "Effective Obs. (Indivs. 2-Person Fams)" is the equivalent number of individuals in 2-person switching families that provide the same amount of variation as switchers. Both of these are calculated using Equation 3, where the denominator is the variance of Head Start, residualized by the family mean of the covariates in the analysis, or 0.125 , respectively. Panel B shows the weighted average of the coefficients when using regression weights, $\omega_{z}$ (defined in Section 3), determined by the overall distribution of families ("All"), the distribution of $2+$ child families ("Siblings"), and the distribution of $2+$ child families that have variation in Head Start attendance ("Switchers"). * $\mathrm{p}<.10,{ }^{* *} \mathrm{p}<.05,{ }^{* * *} \mathrm{p}<.01$. Sources: Panel Study of Income Dynamics, 1968-2011 waves and Children of the National Longitudinal Study of Youth. 
Table 5: Monte Carlo Experiments: Bias of Reweighting and FFE Relative to True ATE, and Efficiency of Reweighting Relative to FFE

\begin{tabular}{|c|c|c|c|c|}
\hline & \multirow[b]{2}{*}{ True ATE } & \multicolumn{2}{|c|}{ Bias: } & \multirow{2}{*}{$\frac{\text { Ratio: MSE of Reweight to }}{\text { MSE of FE }}$} \\
\hline & & $\mathrm{FE}$ & Reweight & \\
\hline \multicolumn{5}{|c|}{ A. Constant TE; p-score: $X_{i g}$} \\
\hline Switchers & 80 & -0.3 & -0.2 & 1.03 \\
\hline Siblings & 80 & -0.3 & -0.5 & 1.19 \\
\hline All & 80 & -0.3 & -0.5 & 1.20 \\
\hline HS Participants & 80 & -0.3 & -0.3 & 1.04 \\
\hline \multicolumn{5}{|c|}{ B. Large family TE; p-score: large family } \\
\hline Switchers & 83.0 & $-11.1^{*}$ & -0.6 & 0.92 \\
\hline Siblings & 49.6 & $22.2^{*}$ & -0.1 & 0.70 \\
\hline All & 40.3 & $31.6^{*}$ & 0.1 & 0.54 \\
\hline HS Participants & 41.1 & $30.7^{*}$ & 0.1 & 0.55 \\
\hline \multicolumn{5}{|c|}{ C. TE linear in $X_{i g} ; p$-score: $X_{i g}$} \\
\hline Switchers & 94.2 & $-2.0^{*}$ & -0.6 & 1.03 \\
\hline Siblings & 80.1 & $12.2^{*}$ & $1.6^{*}$ & 0.99 \\
\hline All & 80.0 & $12.2^{*}$ & $1.7^{*}$ & 1.00 \\
\hline HS Participants & 91.5 & 0.8 & -0.2 & 1.03 \\
\hline \multicolumn{5}{|c|}{ D. TE linear in $X_{i g} ; p$-score: $X_{i g}$ spline } \\
\hline Switchers & 94.2 & $-1.5^{*}$ & -0.3 & 1.04 \\
\hline Siblings & 80.1 & $12.7^{*}$ & -0.4 & 1.08 \\
\hline All & 80.0 & $12.8^{*}$ & -0.4 & 1.09 \\
\hline HS Participants & 91.5 & 1.3 & -0.2 & 1.09 \\
\hline
\end{tabular}

Notes: This table shows the results from 3,000 Monte Carlo simulations. Each panel of the table shows results from a different DGP and/or different covariates used in the p-score, and each row within panel is for a different target population. The true DGP is linear, and is discussed in Section 4.4. The first panel shows results where Head Start has a constant treatment effect (TE) for all individuals; the second shows results where Head Start (HS) has no effect on individuals from small families (3 or fewer children) and a large effect for families with many children (4 or more children); and the third and fourth panels show results where treatment effects that are linear in $X_{i g}$. Column 1, "True Beta," presents the true average increase in the probability of completing some college for participants in Head Start in the sample, which is a function of the DGP and sample composition. Columns 2 and 3 present the bias of various estimation strategies, defined as the difference between the estimated effects of Head Start and the true beta. The estimated effects come from a LPM, propensity-score weighted LPM, respectively. Column 4 presents the ratio of the mean squared error (MSE) of the reweighting estimators relative to LPM. Reweighted estimates are obtained using in-regression weighting, with weights adjusting for the representativeness of switchers (using the variable(s) indicated in each of the panel headings as predictors in the multinomial logit step) and the conditional variance of Head Start within families. All betas are multiplied by 1,000. ${ }^{*} \mathrm{p}<.01$. 
Table 6: Head Start Impact for Representative Eligible Children, Participants, and Siblings

Using Reweighting

\begin{tabular}{|c|c|c|c|c|c|c|}
\hline & \multicolumn{2}{|c|}{ FFE } & \multicolumn{3}{|c|}{ Reweighted ATE, Target $=$} & \multirow{2}{*}{$\begin{array}{c}\text { Diff. b/w } \\
\text { FFE and } \\
\text { Participant ATE }\end{array}$} \\
\hline & GTC/Deming & $\begin{array}{c}\text { Expand Sample/ } \\
\text { Replicate }\end{array}$ & HS Eligible & Participants & Siblings & \\
\hline \multicolumn{7}{|c|}{ A. Some College (PSID) } \\
\hline Head Start & $\begin{array}{l}0.281^{* *} \\
(0.108)\end{array}$ & $\begin{array}{l}0.120^{* *} \\
(0.053)\end{array}$ & $\begin{array}{c}0.052 \\
(0.064)\end{array}$ & $\begin{array}{c}0.021 \\
(0.059)\end{array}$ & $\begin{array}{c}0.064 \\
(0.061)\end{array}$ & $\begin{array}{l}0.099^{* *} \\
(0.032)\end{array}$ \\
\hline Y Mean in Target & - & 0.556 & 0.387 & 0.437 & 0.556 & \\
\hline \multicolumn{7}{|c|}{ B. Economic Sufficiency Index, Age 30 (PSID) } \\
\hline Head Start & - & $\begin{array}{l}-0.023 \\
(0.102)\end{array}$ & $\begin{array}{l}-0.071 \\
(0.101)\end{array}$ & $\begin{array}{l}-0.040 \\
(0.099)\end{array}$ & $\begin{array}{c}0.021 \\
(0.113)\end{array}$ & $\begin{array}{c}0.017 \\
(0.066)\end{array}$ \\
\hline Y Mean in Target & - & 0.213 & -0.198 & -0.485 & 0.213 & \\
\hline \multicolumn{7}{|c|}{ C. High School Graduation (CNLSY) } \\
\hline Head Start & $\begin{array}{c}0.086^{* * *} \\
(0.031)\end{array}$ & $\begin{array}{c}0.085^{* * *} \\
(0.030)\end{array}$ & $\begin{array}{c}0.033 \\
(0.042) \\
\end{array}$ & $\begin{array}{c}0.048 \\
(0.037) \\
\end{array}$ & $\begin{array}{c}0.020 \\
(0.044) \\
\end{array}$ & $\begin{array}{l}0.037^{*} \\
(0.023)\end{array}$ \\
\hline Y Mean in Target & - & 0.776 & 0.734 & 0.766 & 0.776 & \\
\hline \multicolumn{7}{|l|}{ D. Idle (CNLSY) } \\
\hline$\overline{\text { Head Start }}$ & $\begin{array}{l}-0.071^{*} \\
(0.038)\end{array}$ & $\begin{array}{l}-0.072^{*} \\
(0.037)\end{array}$ & $\begin{array}{l}-0.061 \\
(0.045)\end{array}$ & $\begin{array}{l}-0.055 \\
(0.042)\end{array}$ & $\begin{array}{l}-0.067 \\
(0.050)\end{array}$ & $\begin{array}{l}-0.017 \\
(0.026)\end{array}$ \\
\hline Y Mean in Target & - & 0.197 & 0.221 & 0.201 & 0.197 & \\
\hline \multicolumn{7}{|c|}{ E. Learning Disability (CNLSY) } \\
\hline Head Start & $\begin{array}{c}-0.059^{* * *} \\
(0.020)\end{array}$ & $\begin{array}{c}-0.059^{* * *} \\
(0.021)\end{array}$ & $\begin{array}{l}-0.031 \\
(0.026)\end{array}$ & $\begin{array}{l}-0.042^{*} \\
(0.022)\end{array}$ & $\begin{array}{l}-0.040 \\
(0.026)\end{array}$ & $\begin{array}{c}0.017 \\
(0.015)\end{array}$ \\
\hline Y Mean in Target & - & 0.051 & 0.055 & 0.041 & 0.051 & \\
\hline \multicolumn{7}{|c|}{ F. Poor Health (CNLSY) } \\
\hline Head Start & $\begin{array}{c}-0.070^{* * *} \\
(0.026)\end{array}$ & $\begin{array}{c}-0.069^{* * *} \\
(0.026)\end{array}$ & $\begin{array}{l}-0.063^{*} \\
(0.037)\end{array}$ & $\begin{array}{c}-0.067^{* *} \\
(0.034)\end{array}$ & $\begin{array}{l}-0.050 \\
(0.038)\end{array}$ & $\begin{array}{l}-0.003 \\
(0.020)\end{array}$ \\
\hline Y Mean in Target & - & 0.103 & 0.098 & 0.074 & 0.103 & \\
\hline
\end{tabular}

Notes: Column 1 of this table shows the FFE estimated impacts of Head Start for whites from GTC or for the whole sample from Deming (2009). Column 2 shows the FFE estimate using our expanded sample for PSID outcomes and using our replication sample for CNLSY outcomes. The outcomes in Panels A and B are taken from the PSID white sample, and the outcomes in Panels C to F are taken from the CNLSY sample. Columns 3 to 5 present reweighted estimates of the effect of Head Start for three target populations (shown in the column header) using the post-regression reweighting procedure, in which we multiply group-level estimates of the impact of Head Start by the representative weight for the target population of interest. Column 6 presents the difference in the estimate in column 2 (FFE) and column 4 (reweighted for participants). "-" is used to indicate that the information is not available. Sample size is $\mathrm{N}=2,986$ for the expanded sample, and 1,036 for GTC. Standard errors obtained by bootstrapping. ${ }^{*} \mathrm{p}<.10, * * \mathrm{p}<.05,{ }^{* * *} \mathrm{p}<$ 01 